\title{
СИНТЕЗ И ИССЛЕДОВАНИЕ АЛГОРИТМОВ УПРАВЛЕНИЯ ДВУХМАССОВОЙ СИСТЕМОЙ С ДВИГАТЕЛЕМ ПОСТОЯННОГО ТОКА
}

\section{SYNTHESIS \& RESEARCH \\ FOR ALGORITHMS OF CONTROL \\ FOR DOUBLE-MASS SYSTEM WITH CONSTANT CURRENT ENGINE}

\section{K. Voloshinovsky}

Summary. Article dedicated to subjects of developing systems with asymptotic characteristics \& makes attach them to linearized systems with infinite or half-infinite settled margin supply reserve. High dynamical accuracy systems and just a little bit sensitive to control object parameters changing exposed to theoretically and with mathematical modeling application due to analytically examine opportunity for automatic systems algorithmic support. Concerned the method of backreturn dynamics tasks parameter adaptive algorithms for automatic systems control with electromechanical double-mass system. Back-return dynamics task method can be applied not only for linear systems, and in addition hold on a comparison possibility with logarithmic amplitude phase frequency characteristics (LAFC).

Keywords: DC motor, control algorithms, automatic systems, parameters of controlled objects.

\author{
Волошиновский Кирилл Иванович \\ К.т.н., дочент, Национальный исследовательских \\ технологический университет МИСиС \\ gas7dev@gmail.com
}

Аннотация. В статье рассматриваются вопросы построения систем с асимптотическими частотными характеристиками, которые придают линейной системе бесконечный или полубесконечный запас устойчивости. Теоретически и методами математического моделирования проводится исследование возможности аналитического проектирования алгоритмического обеспечения автоматических систем, обладающих высокой динамической точностью и слабой параметрической чувствительностью к изменениям параметров управляемых объектов. Рассматривается синтез методом обратных задач динамики параметрически адаптивных алгоритмов автоматического управления электромеханической двухмассовой системой. Методы обратных задач динамики могут применяться не только для линейных систем, а результаты их применения, полученные для линейных систем можно сопоставить с анализом методом логарифмических амплитудно-фазочастотных характеристик (ЛАФЧХ).

Ключевые слова: двигатель постоянного тока, алгоритмы управления, автоматические системы, параметры управляемых объектов.

приводит к другим моделям систем рассматриваемых совместно с алгоритмом управления. Таким образом, можно свести обе обмотки электрической машины к общему входу $u_{\text {упр. }}$

В процессе моделирования результатов синтеза мы наблюдаем устойчивые процессы, полученные в процессе пошаговых расчетов численными методами, что приводит к предположению о существовании универсальных методов адаптации аналоговых алгоритмов прототипов и их преобразованию в цифровую форму с сохранением динамический свойств. Для адаптации к условиям квантования дискретных систем необходимо предложить квадратурные формулы звеньев, т.е. их дискретные изображения, например по Лапласу, в т.ч. звеньев интегрирования, удобные для цифрового моделирования, а также для работы полученных алгоритмов в составе микропроцессорных систем управления.

Аналоговую систему можно либо включить непосредственно в состав цифровой и получить «комбинированную» систему, или превратить аналоговый прототип в цифровой алгоритм, что является колоссальным преи- 

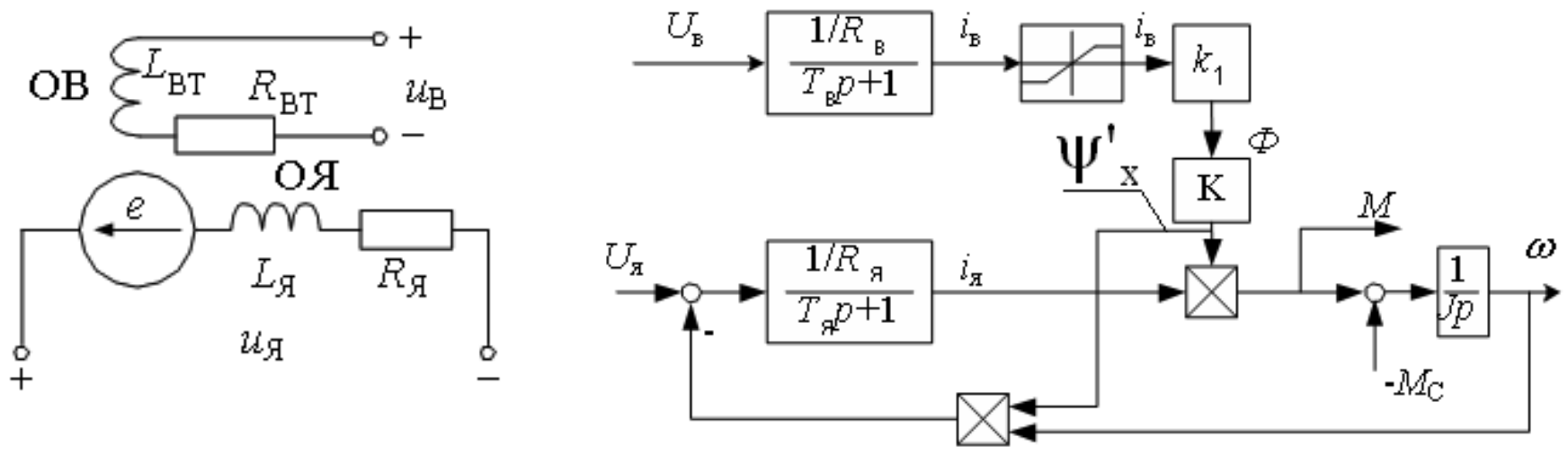

Рис. 1. Двигатель постоянного тока (ДПТ) с обмоткой возбуждения

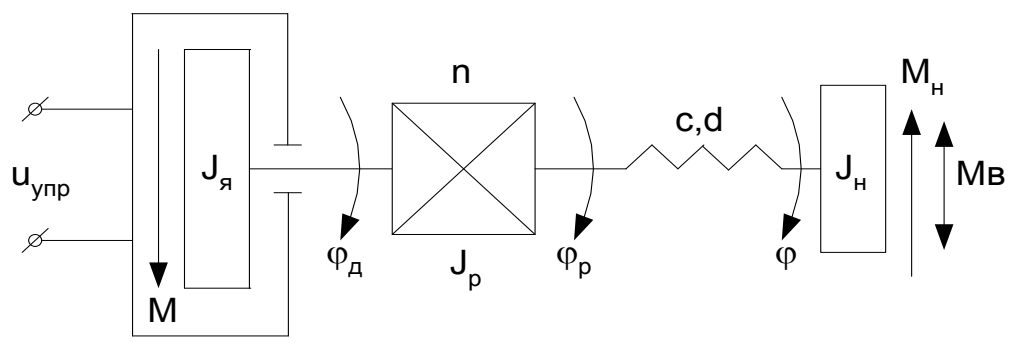

Рис. 2. Физическая модель двухмассовой системы с дПт

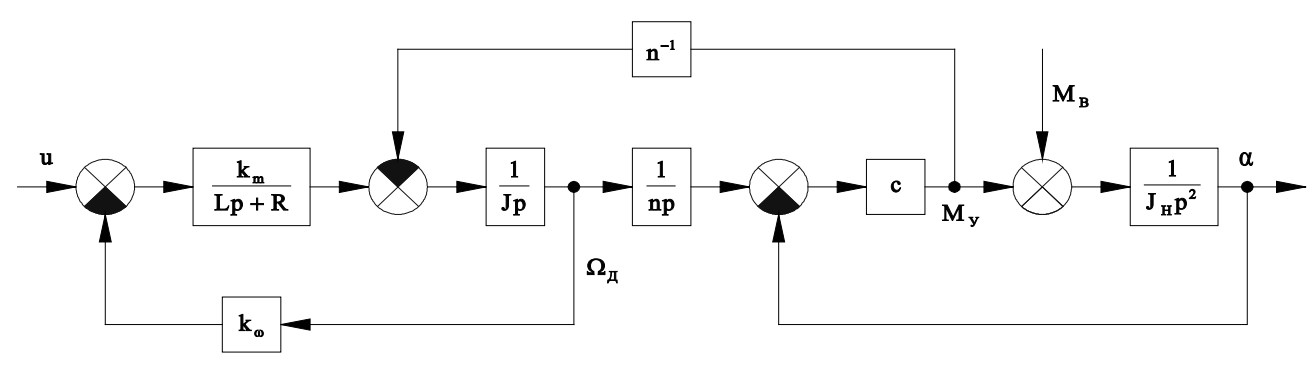

Рис. 3. Структурная схема математической модели упругой двухмассовой системы

муществом аналоговых систем, особенно проверенных. Обратный переход от цифрового алгоритма к аналоговому прототипу осложнен. Поэтому имеет смысл удостоить вниманием именно методы синтеза непрерывных аналоговых систем.

\section{1. Физическал модель Авухмассовой системы}

На рисунке 2 приведена схема физической модели двухмассовой системы с упругой механической передачей движения. Система состоит из исполнительного двигателя, редуктора с коэффициентом передачи $n$ и нагрузки. Свойства конструкции редуктора характеризуются коэффициентом упругости с[Н·м · рад $\left.^{-1}\right]$ и жесткости муфты d. Моменты инерции якоря исполнительного двигателя (ДПТ), редуктора и нагрузки обозначены соответственно - $\mathrm{J}_{\mathrm{a}}, \mathrm{J}_{\mathrm{D}}, \mathrm{J}_{\mathrm{u}}$. Угол поворота вала и нагрузки обозначены соответственно $\varphi_{д}$, $\varphi$. К нагрузке приложен возмущающий момент $\mathrm{M}_{\mathrm{B}}$. Управляющим сигналом является напряжение якорной цепи $u$.

\section{2. Структурная схема математической моде^и}

Структурная схема математической модели двухмассовой системы с упругой передачей движения, исполнительным элементом которой является двигатель постоянного тока независимого возбуждения приведена на рисунке 3. 
Таблица 1. Параметры физической модели объекта управления (ОУ)

\begin{tabular}{|c|c|c|c|c|}
\hline $\mathbf{J}$ & $\mathbf{J}_{\mathbf{H}}$ & $\mathbf{n}$ & $\mathbf{L}$ & $\mathbf{R}$ \\
\hline $2,94 \times 10^{-4} \mathrm{\kappa} \Gamma \times \mathrm{M}^{3}$ & $6 \mathrm{\kappa} \Gamma \times \mathrm{M}^{2}$ & $10^{2}$ & $10^{-3} \Gamma_{\mathrm{H}}$ & 3,8 Ом \\
\hline $\mathbf{k}_{\mathrm{m}}$ & \multicolumn{2}{|c|}{$\mathbf{k}_{\Omega}$} & \multicolumn{2}{|c|}{ c } \\
\hline $0,043 \mathrm{H} \times \mathrm{M} \times \mathrm{A}^{-1}$ & \multicolumn{2}{|c|}{$0,073 \mathrm{~B} \times \mathrm{c} \times$ рад $^{-1}$} & \multicolumn{2}{|c|}{$3 \times 105 \mathrm{H} \times \mathrm{M} \times$ рад $^{-1}$} \\
\hline
\end{tabular}

Таблица 2. Полюсы передаточной функции объекта управления при различных значениях момента инерции нагрузки

\begin{tabular}{|c|c|c|c|}
\hline $\mathbf{J}_{\mathbf{H}}$ & \multicolumn{2}{|c|}{$\mathbf{p}_{\mathbf{1 , 2}}$} & $\mathbf{p}_{3, \mathbf{4}}$ \\
\hline 6 & \multicolumn{2}{|c|}{$-47,784 \pm 32,795 i$} & $-2,216 \pm 398,66 i$ \\
\hline 30 & $-81,64$ & $-10,796$ & $-3,782 \pm 348,027 i$ \\
\hline
\end{tabular}

Динамические свойства двигателя характеризуются параметрами $\mathrm{k}_{\mathrm{m}}, \mathrm{k}_{\Omega}$, индуктивностью L и активным сопротивлением якорной цепи. Суммарный момент инерции якоря редуктора в пересчете к валу двигателя обозначен через $\mathrm{J}_{\text {д }}=\mathrm{J}_{\mathrm{g}}+\mathrm{J}_{\mathrm{P}} / \mathrm{n}^{2}$. Момент, обусловленный упругой деформацией, обозначен $\mathrm{M}_{\mathrm{y}}$. K нагрузке приложен возмущающий момент $\mathrm{M}_{\mathrm{B}}$ (см. рис. 3). Математическую модель управляемой системы будем рассматривать с числовыми значениями параметров, отвечающими заданию на проектирование, принимая их равными (табл. 1):

\section{3. Перелаточная функция объекта управления}

Приведенная схема двухмассовой системы соответствует передаточной функции (ПФ ОУ):

$$
\begin{aligned}
& \mathrm{W}_{0}^{\prime}(\mathrm{p})=\frac{\alpha(\mathrm{p})}{\mathrm{u}(\mathrm{p})}=\frac{\mathrm{b}_{0}^{\prime}}{\mathrm{p}^{5}+\mathrm{a}_{4}^{\prime} \mathrm{p}^{4}+\ldots+\mathrm{a}_{1}^{\prime} \mathrm{p}+\mathrm{a}_{0}^{\prime}}=\frac{\mathrm{N}}{\mathrm{A}(\mathrm{p})} \\
& \mathrm{A}(\mathrm{p})=\mathrm{p}\left[\mathrm{J}_{\mathrm{H}} \mathrm{n}^{2} J \mathrm{Lp}^{4}+\mathrm{J}_{\mathrm{H}} \mathrm{n}^{2} J R \mathrm{p}^{3}+\left(\mathrm{cLJ} \mathrm{H}_{\mathrm{H}}+\mathrm{cn}^{2} J \mathrm{~L}+\mathrm{J}_{\mathrm{H}} \mathrm{n}^{2} \mathrm{k}_{\mathrm{m}} \mathrm{k}_{\Omega}\right) \mathrm{p}^{2}+\right. \\
& \left.+\mathrm{c}\left(\mathrm{RJ}_{\mathrm{H}}+\mathrm{n}^{2} \mathrm{JR}\right) \mathrm{p}+\mathrm{cn}^{2} \mathrm{k}_{\mathrm{m}} \mathrm{k}_{\Omega}\right]
\end{aligned}
$$

$$
\mathrm{N}=\mathrm{ck}_{\mathrm{m}} \mathrm{n}
$$

Указанная функция $\mathrm{W}_{0}^{\prime}(\mathrm{p})$ обладает астатизмом первого порядка:

$$
\begin{aligned}
& \mathrm{M}(\mathrm{p})=\mathrm{m}_{5} \mathrm{p}^{5}+\mathrm{m}_{4} \mathrm{p}^{4}+\mathrm{m}_{3} \mathrm{p}^{3}+\mathrm{m}_{2} \mathrm{p}^{2}+\mathrm{m}_{1} \mathrm{p}+\mathrm{m}_{0} \\
& \mathrm{~m}_{5}=\mathrm{J}_{\mathrm{H}} \mathrm{n}^{2} \mathrm{JL} ; \mathrm{m}_{4}=\mathrm{J}_{\mathrm{H}} \mathrm{n}^{2} \mathrm{JR} ; \\
& \mathrm{m}_{3}=\mathrm{cLJ}_{\mathrm{H}}+\mathrm{cn}^{2} \mathrm{JL}+\mathrm{J}_{\mathrm{H}} \mathrm{n}^{2} \mathrm{k}_{\mathrm{m}} \mathrm{k}_{\Omega} \\
& \mathrm{m}_{1}=\mathrm{cn}^{2} \mathrm{k}_{\mathrm{m}} \mathrm{k}_{\Omega} ; \mathrm{m}_{0}=0 \Rightarrow \text { астатизм первого порядка }
\end{aligned}
$$

Приведем $\mathrm{W}_{0}^{\prime}(\mathrm{p})$ к каноническому виду:

$$
\mathrm{b}_{0}^{\prime}=\mathrm{N} / \mathrm{m}_{5}
$$

$$
\mathrm{a}_{4}^{\prime}=\mathrm{m}_{4} / \mathrm{m}_{5} ; \mathrm{a}_{3}^{\prime}=\mathrm{m}_{3} / \mathrm{m}_{5} ; \mathrm{a}_{2}^{\prime}=\mathrm{m}_{2} / \mathrm{m}_{5} ; \mathrm{a}_{1}^{\prime}=\mathrm{m}_{1} / \mathrm{m}_{5} ; \mathrm{a}_{0}^{\prime}=0(7)
$$

Полюсы передаточной функции при различных значениях момента инерции нагрузки приведены в таблице 2.

При $\mathrm{L}=0$ ，

$$
\mathrm{W}_{0}(\mathrm{p})=\frac{\alpha(\mathrm{p})}{\mathrm{u}(\mathrm{p})}=\frac{\mathrm{b}_{0}}{\mathrm{p}^{4}+\mathrm{a}_{3} \mathrm{p}^{3}+\ldots+\mathrm{a}_{1} \mathrm{p}+\mathrm{a}_{0}}=\frac{\mathrm{N}}{\mathrm{M}(\mathrm{p})}
$$

Дифференциальное уравнение объекта управления на основе пропорции отношения входа к выходу ПФ ОУ в операторной форме запишем в виде:

$$
\dddot{\alpha}=\frac{\mathrm{b}_{0}}{\mathrm{p}}-\alpha \frac{\mathrm{A}^{\prime}(\mathrm{p})}{\mathrm{p}},
$$

где

$$
\mathrm{A}^{\prime}(\mathrm{p})=\sum_{\mathrm{i}=1}^{3} \mathrm{a}_{\mathrm{i}} \mathrm{p}^{\mathrm{i}},
$$

p — оператор дифференцирования.

\section{4. А^горитм управ^ения угловым положением с обратной связью по ускорению, исслеАование $\triangle$ инамики системы}

\section{1. Требования к системе управления, эталонная модель.}

Выберем такую систему управления, переходной процесс, в которой удовлетворял бы поставленным в задаче требованиям. Для этого исследуем следующую передаточную функцию.

$$
\mathrm{W}_{\mathrm{M}}(\mathrm{p})=\frac{\mathrm{a}_{0} \mathrm{p}^{2}+\mathrm{a}_{1} \mathrm{p}+\mathrm{a}_{2}}{\mathrm{p}\left(\mathrm{p}^{2}+\breve{\mathrm{b}}_{1} \mathrm{p}+\breve{\mathrm{b}}_{2}\right)}
$$

Последовательное включение одного или двух апериодических звеньев с параметром $\tau \approx 0,0033$ (порядка 
Таблица 3. Полюсы ПФ замкнутой эталонной модели

\begin{tabular}{|l|l|l|}
\hline$p_{1}$ & $p_{2}$ & $p_{3,4}$ \\
\hline$-70,242$ & $-4,664$ & $-12,797 \pm$ \\
\hline
\end{tabular}

данной величины) практически не влияет на характер переходного процесса.

$$
\begin{aligned}
& \mathrm{W}_{\mathrm{M} 1}(\mathrm{p})=\frac{\mathrm{a}_{0} \mathrm{p}^{2}+\mathrm{a}_{1} \mathrm{p}+\mathrm{a}_{2}}{\mathrm{p}\left(\mathrm{p}^{2}+\breve{\mathrm{b}}_{1} \mathrm{p}+\breve{\mathrm{b}}_{2}\right)} \times \frac{1}{(\tau \mathrm{p}+1)}=\frac{\xi}{\mathrm{u}} \\
& \mathrm{W}_{\mathrm{M} 2}(\mathrm{p})=\frac{\mathrm{a}_{0} \mathrm{p}^{2}+\mathrm{a}_{1} \mathrm{p}+\mathrm{a}_{2}}{\mathrm{p}\left(\mathrm{p}^{2}+\breve{\mathrm{b}}_{1} \mathrm{p}+\breve{\mathrm{b}}_{2}\right)} \times \frac{1}{(\tau p+1)^{2}}=\frac{\xi}{\mathrm{u}} \\
& \mathrm{p}\left(\mathrm{p}^{2}+\breve{\mathrm{b}}_{1} \mathrm{p}+\breve{\mathrm{b}}_{2}\right)(\tau \mathrm{p}+1)=\mathrm{p}\left(\tau \mathrm{p}^{3}+\breve{\mathrm{b}}_{1} \tau \mathrm{p}^{2}+\breve{\mathrm{b}}_{2} \tau \mathrm{p}+\mathrm{p}^{2}+\breve{\mathrm{b}}_{1} \mathrm{p}+\breve{\mathrm{b}}_{2}\right)= \\
& =\tau \mathrm{p}^{4}+\breve{\mathrm{b}}_{1} \tau \mathrm{p}^{3}+\breve{\mathrm{b}}_{2} \tau \mathrm{p}^{2}+\mathrm{p}^{3}+\breve{\mathrm{b}}_{1} \mathrm{p}^{2}+\breve{\mathrm{b}}_{2} \mathrm{p}= \\
& \mathrm{b}_{0}=\tau ; \mathrm{b}_{1}=1+\breve{\mathrm{b}}_{1} \tau ; \mathrm{b}_{2}=\breve{\mathrm{b}}_{1}+\breve{\mathrm{b}}_{2} \tau ; \mathrm{b}_{3}=\breve{\mathrm{b}}_{2}
\end{aligned}
$$

Тогда дифференциальное уравнение для эталонной модели выглядит следующим образом:

$$
\mathrm{b}_{0} \xi^{(4)}+\mathrm{b}_{1} \xi^{(3)}+\mathrm{b}_{2} \xi^{(2)}+\mathrm{b}_{3} \xi^{(1)}=\mathrm{a}_{0} \varphi_{\mathrm{Bx}}^{(2)}+\mathrm{a}_{1} \varphi_{\mathrm{Bx}}^{(1)}+\mathrm{a}_{1} \varphi_{\mathrm{BX}}
$$

Пусть требованиям задания на проектирование по динамической точности, быстродействию и другим показателям качества процесса отвечает эталонная модель, описываемая передаточной функцией разомкнутой системы. Передаточная функция эталонной модели имеет вид:

$$
\begin{aligned}
& \mathrm{W}_{\mathrm{M}}(\mathrm{p})=\frac{80(0,3 \mathrm{p}+1)}{\mathrm{p}(2 \mathrm{p}+1)(0,02 \mathrm{p}+1)(\tau \mathrm{p}+1)}\left(\frac{1}{\tau} \approx 5 \varpi_{\mathrm{cp}}^{*}, \tau \cong 0,02\right) \\
& \mathrm{W}_{\mathrm{M}}(\mathrm{p})=\frac{80(0,3 \mathrm{p}+1)}{\mathrm{p}(2 \mathrm{p}+1)(0,02 \mathrm{p}+1)^{2}}
\end{aligned}
$$

Длительность переходного процесса: $\mathrm{t}_{\Pi}=0,6 \mathrm{c}$ .Величина перерегулирования: $\sigma=23 \%$. Динамическая точность $\varepsilon=0,3 \cdot 10^{-3}$ рад. Соответствующая эталонной модели замкнутая система имеет ПФ:

$$
\begin{aligned}
& \mathrm{K}_{\mathrm{M}}(\mathrm{p})=\frac{\mathrm{W}_{\mathrm{M}}(\mathrm{p})}{1+\mathrm{W}_{\mathrm{M}}(\mathrm{p})}=\frac{80(0,3 \mathrm{p}+1)}{\mathrm{p}(2 \mathrm{p}+1)(0,02 \mathrm{p}+1)^{2}+80(0,3 \mathrm{p}+1)}= \\
& =\frac{30000 \mathrm{p}+100000}{\mathrm{p}^{4}+100,5 \mathrm{p}^{3}+2550 \mathrm{p}^{2}+31250 \mathrm{p}+100000}
\end{aligned}
$$

Примем обозначения соответствующих коэффициенTOB.

$\beta_{1}=30000 \quad \beta_{0}=100000 \quad \gamma_{3}=100,5 \quad \gamma_{2}=2550 \quad \gamma_{1}=31250$ $\gamma_{0}=100000$
Передаточная функция замкнутой эталонной модели примет вид:

$$
\mathrm{K}_{\mathrm{M}}(\mathrm{p})=\frac{\beta_{1} \mathrm{p}+\beta_{0}}{\mathrm{p}^{4}+\gamma_{3} \mathrm{p}^{3}+\gamma_{2} \mathrm{p}^{2}+\gamma_{1} \mathrm{p}+\gamma_{0}}=\frac{\alpha_{\mathrm{M}}}{\alpha_{\mathrm{BX}}}
$$

Полюсы передаточной функции замкнутой эталонной модели приведены в таблице 3.

\section{2. Асимптотическая логарифмическая характеристика эталонной модели}

Годограф, логарифмические амплитудно-частотные и фазочастотные характеристики, переходной процесс замкнутой эталонной модели приведены на рисунке 4 (белым), в сравнении с ЛАФЧХ двухмассовой системы при разных значениях момента инерции нагрузки (черным).

Частота среза определится в месте пересечения ЛАЧХ оси (координат) частот. Запас устойчивости определится как длина отрезка между частотой среза и точкой пересечения фазовой характеристики оси $-\pi / 2$ радиан. ФЧХ двухмассовой системы (черным) содержит точку разрыва второго рода, которую требуется устранить в процессе синтеза, для получения монотонной характеристики. Таким образом, исходя из метода ЛАФЧХ эталонная модель обладает бесконечным запасом устойчивости. Дифференциальное уравнение, которым описывается эталонная модель:

$$
\alpha_{M}^{(4)}=\beta_{1} \dot{\alpha}_{B X}+\beta_{0} \alpha_{B X}-\gamma_{0} \alpha_{M}-\gamma_{1} \dot{\alpha}_{M}-\gamma_{2} \ddot{\alpha}_{M}-\gamma_{3} \ddot{\alpha}_{M}(20)
$$

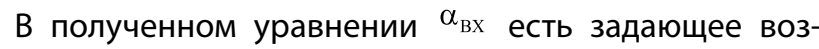
действие, которое должна отрабатывать, т.е. воспроизводить проектируемая система с требуемой точностью:

$$
\left|\alpha_{B X}(t)-\alpha(t)\right| \leq \varepsilon
$$

По построению эталонной модели с необходимостью выполняется неравенство:

$$
\left|\alpha_{M}(t)-\alpha(t)\right| \leq \varepsilon
$$

В таком случае определение структуры и параметров алгоритма управления можно выполнять из условия приближения процесса $\varphi(t) \rightarrow \varphi_{\text {вх }}(\mathrm{t})$, с такой точностью при которой достигается равенство $\left|\alpha_{\mathrm{BX}}(\mathrm{t})-\alpha(\mathrm{t})\right|=\varepsilon-\varepsilon^{\prime}$. 

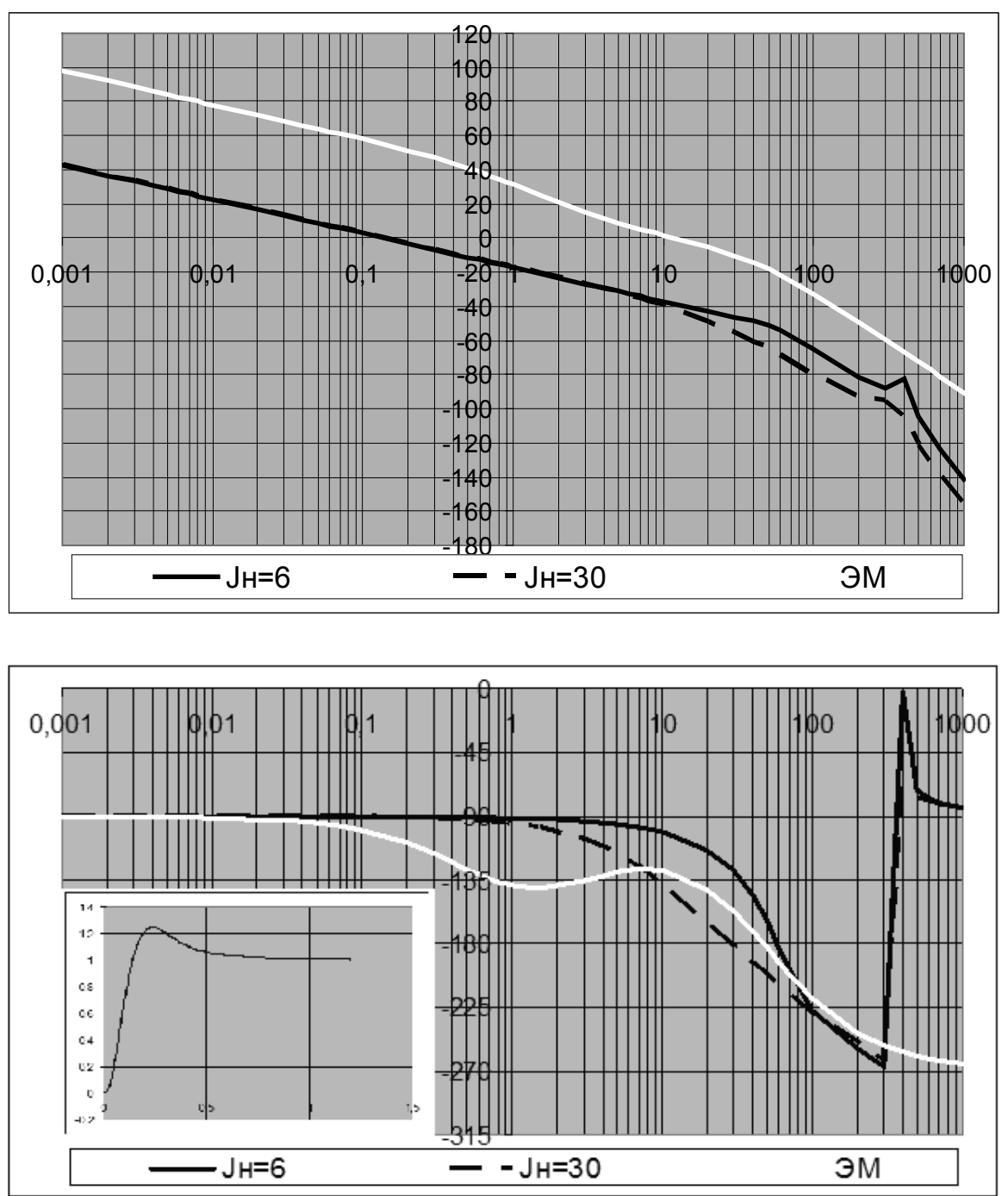

Рис. 4. ЛАФЧХ ОУ и эталонной модели в сравнении

\section{3. Синтез алгоритма управления}

Найдем закон управления из условия предельного приближения переходного процесса в проектируемой системе к переходному процессу в эталонной модели:

$$
\left|\alpha(t)-\alpha_{M}(t)\right| \rightarrow \min
$$

От выражения с модулем, перейдем к минимизации функционала, по градиентной схеме поиска экстремума первого порядка, рассматривая задачу гашения нормированного значения энергии ускорения, вычисляемой в окрестности траектории движения эталонной модели:

$$
\mathrm{G}(\mathrm{u})=\frac{1}{2}\left[\ddot{\alpha}_{\mathrm{M}}(\mathrm{t})-\ddot{\alpha}(\mathrm{t}, \mathrm{u})\right]^{2}=\mathrm{G}(\mathrm{u}) \rightarrow \min , \text { при } \mathrm{t} \rightarrow \infty
$$

Управляющую функцию определим с помощью уравнения:

$$
\frac{\mathrm{du}(\mathrm{t})}{\mathrm{dt}}=\lambda \frac{\mathrm{dG}(\mathrm{u})}{\mathrm{du}}, \lambda=\mathrm{const},
$$

Закон управление запишем в виде:

$$
\mathrm{u}=\mathrm{k}\left(\ddot{\alpha}_{\mathrm{M}}-\ddot{\alpha}\right)
$$

Особенность алгоритма управления состоит в том, что для вычисления управляющей функции необходимо иметь информацию об ускорении $\ddot{\alpha}$.

Из расчета снятия с выхода системы второй производной, выразим вторую производную $\ddot{\alpha}_{м}$.Уравнение эталонной модели запишем в виде:

$$
\begin{aligned}
& \alpha_{M}^{(4)}=\beta_{1} \dot{\alpha}_{B X}+\beta_{0} \alpha_{B X}-\gamma_{0} \alpha_{M}-\gamma_{1} \dot{\alpha}_{M}-\gamma_{2} \ddot{\alpha}_{M}-\gamma_{3} \dddot{\alpha}_{M} \\
& \dddot{\alpha}_{M}=\gamma_{0} \int_{0}^{t}\left(\alpha_{B X}-\alpha_{M}\right) d t+\beta_{1} \alpha_{B X}-\gamma_{1} \alpha_{M}-\gamma_{2} \dot{\alpha}_{M}-\gamma_{3} \ddot{\alpha}_{M}
\end{aligned}
$$




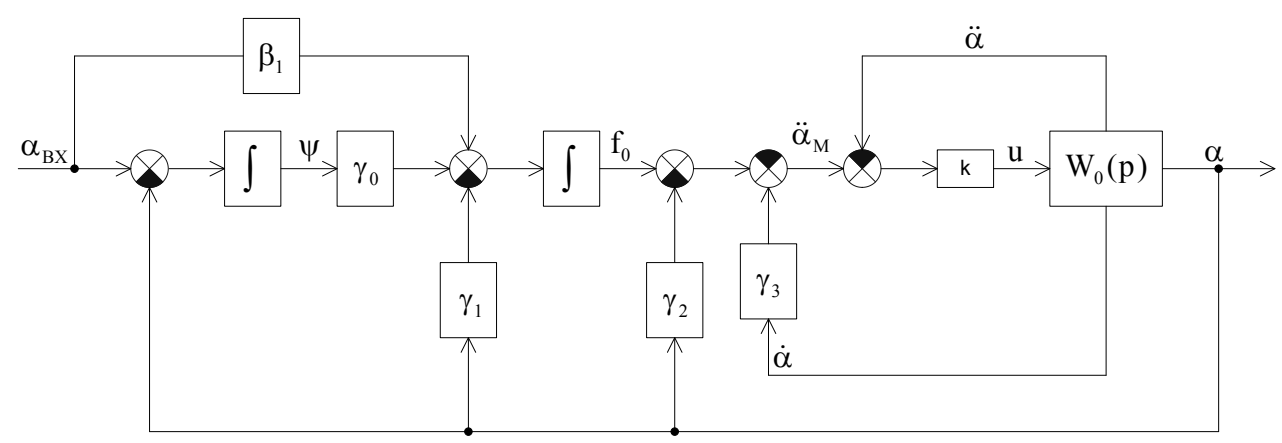

Рис. 5. Синтезированный алгоритм в задаче управления угловым положением с обратной связью по ускорению

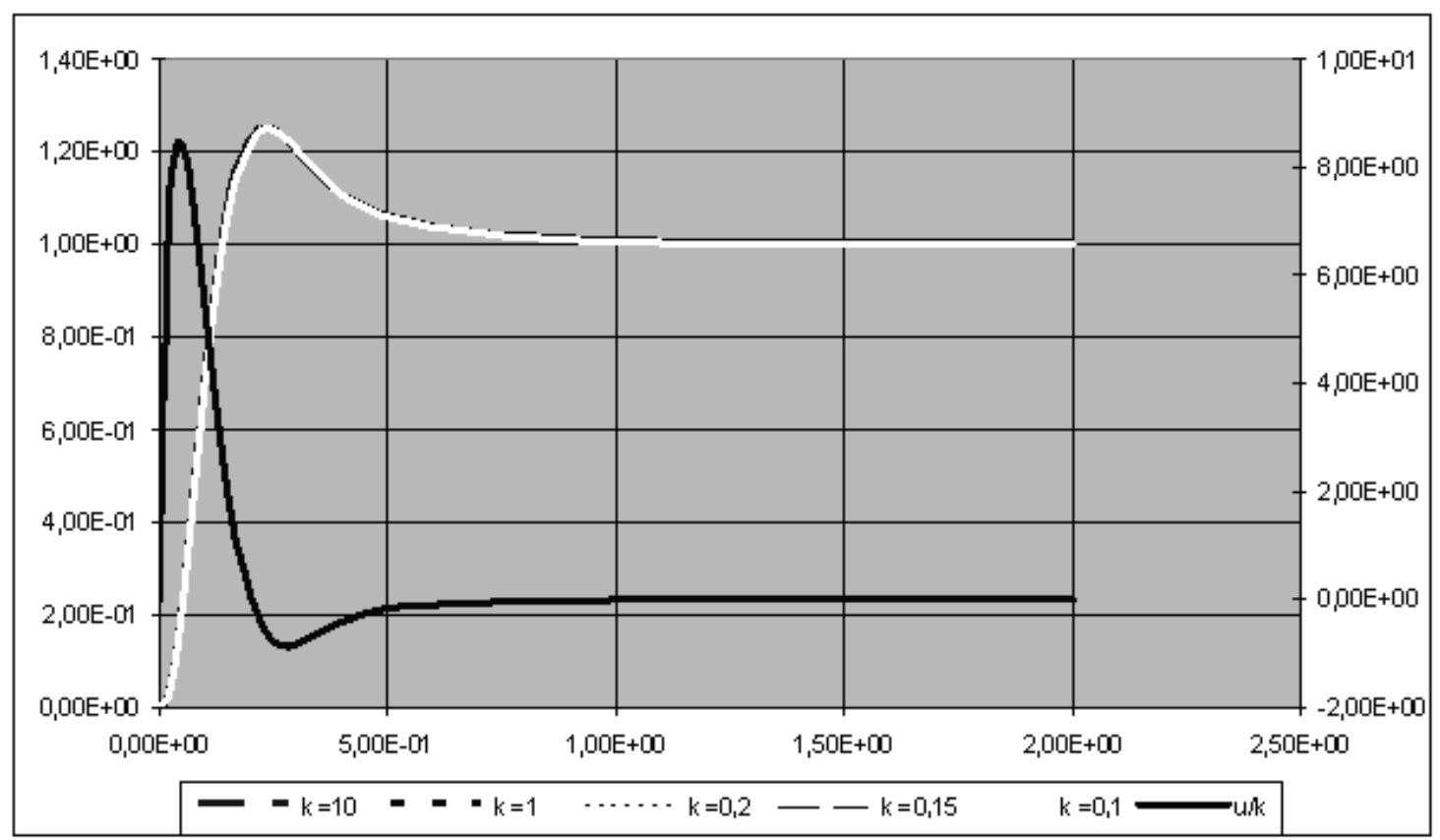

Рис. 6. Переходной процесс и управляющее напряжение при различных значениях настройки k (JH=6)

Введем обозначение:

$\psi_{\mathrm{M}}=\int_{0}^{\mathrm{t}}\left(\alpha_{\mathrm{BX}}-\alpha_{\mathrm{M}}\right) \mathrm{dt}$

Следовательно,

$\ddot{\alpha}_{M}=\int_{0}^{t}\left[\gamma_{0} \psi_{M}+\beta_{1} \alpha_{B X}-\gamma_{1} \alpha_{M}\right] d t-\gamma_{2} \alpha_{M}-\gamma_{3} \dot{\alpha}_{M}$

Алгоритм управления нужно записывать из условия асимптотического приближения $\alpha_{\mathrm{M}} \rightarrow \alpha$.

$\ddot{\alpha}_{\mathrm{M}}=\int_{0}^{\mathrm{t}}\left[\gamma_{0} \psi+\beta_{1} \alpha_{\mathrm{BX}}-\gamma_{1} \alpha\right] \mathrm{dt}-\gamma_{2} \alpha-\gamma_{3} \dot{\alpha}$,

где $\psi=\int_{0}^{\mathrm{t}}\left(\alpha_{\mathrm{Bx}}-\alpha\right) \mathrm{dt}$

$$
\begin{aligned}
& \mathrm{u}=\mathrm{k}\left(\ddot{\alpha}_{M}-\ddot{\alpha}\right) \\
& \dot{\mathrm{u}}=-\lambda \mathrm{b}_{0}\left(\ddot{\alpha}_{M}-\ddot{\alpha}\right) ; \mathrm{k}=-\lambda \mathrm{b}_{0}
\end{aligned}
$$

Уравнения (31)-(33) с учетом выражения (1) для объекта управления, содержат всю необходимую информацию для построения системы управления угловым положением с обратной связью по ускорению (в виде окружения объекта управления) схема которой приводится на рисунке 5.

Критерием технической реализуемости является наличие первой производной в канале обратной связи (ОС). Поэтому проведем синтез с такой же типовой процедурой, но, приводящий к ОС по угловой скорости. 
Таблица 4. Величина перерегулирования при различных значениях момента инерции нагрузки, при

$$
\mathbf{k}=\mathbf{k}^{*}=10
$$

\begin{tabular}{|l|l|l|l|l|l|}
\hline $\mathbf{J}_{\mathbf{H}^{\prime}} \mathbf{K} \boldsymbol{K} \cdot \mathbf{M}^{2}$ & $\mathbf{6}$ & $\mathbf{9}$ & $\mathbf{1 5}$ & $\mathbf{2 0}$ & $\mathbf{3 0}$ \\
\hline$\sigma, \%$ & 23 & 23 & 23 & 23 & 23 \\
\hline
\end{tabular}
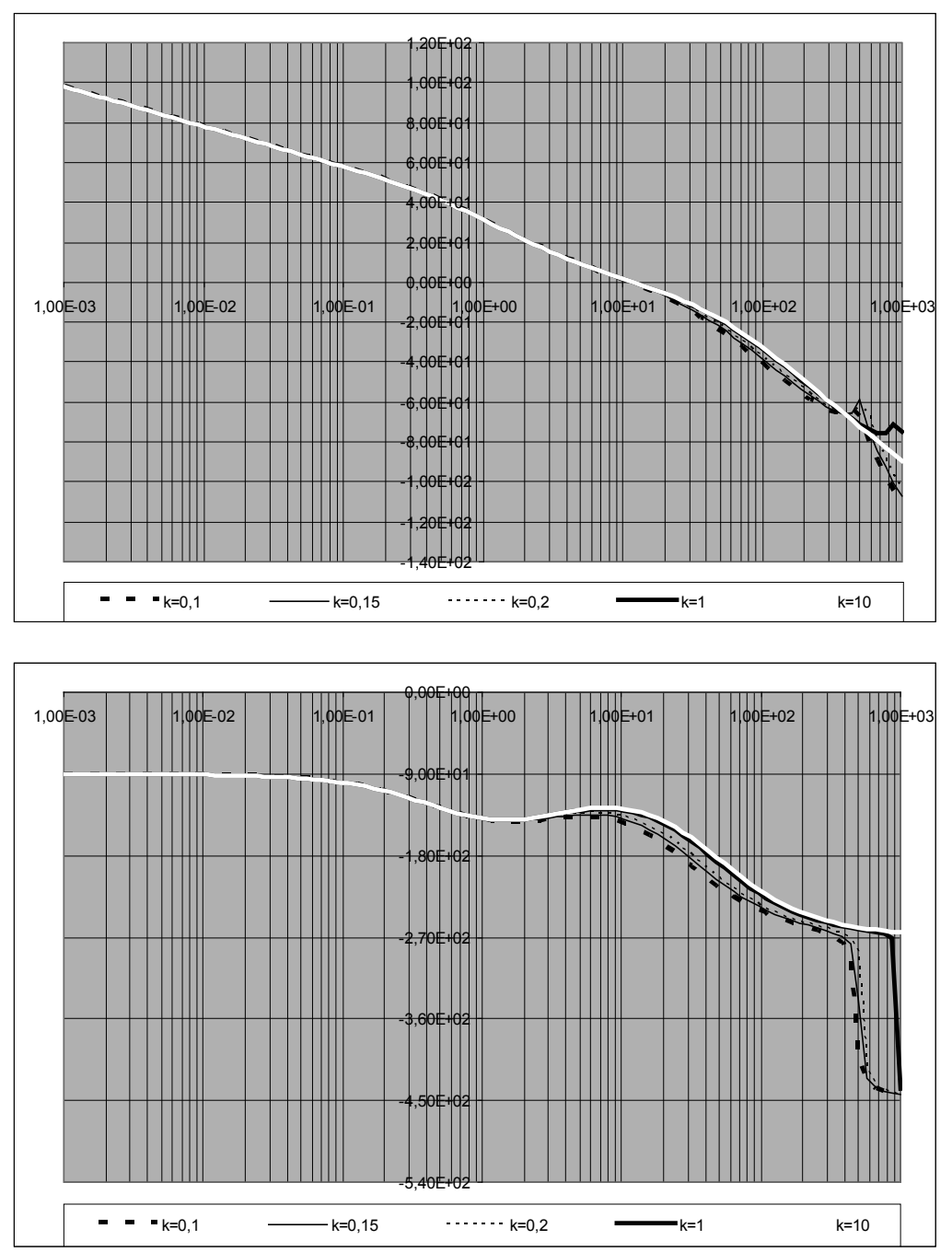

Рис. 7. ЛАЧХ и ЛФЧХ для разомкнутой системы в задаче управления угловым ускорением

\section{4. Исследование динамики замкнутой системы}

Исследуем динамику полученной системы. Прежде всего подберем такой коэффициент усиления при котором переходной процесс асимптотически приближается к эталонному процессу.

На рисунке 6 приводится вид переходного процесса при различных значениях коэффициента усиления при фиксированном значении момента инерции нагрузки, из которого следует, что в диапазоне $\mathrm{k}=0,1 . .10$ динами- ческие характеристики системы практически совпадают с динамическими характеристиками эталонной модели (см. рисунок 4).

Исследуем поведение системы при различных значениях момента инерции нагрузки. Вид переходного процесса приводится на рисунке 8, из которого видно, что в рабочем диапазоне $\mathrm{J}_{\mathrm{H}}=6 \ldots 30$ кг $\mathrm{M}^{2}$ система не чувствительна к изменению момента инерции нагрузки, величина перерегулирования, при этом, остается постоянной и практически полностью совпадает 
Таблица 5. Величина перерегулирования при различных значениях момента инерции нагрузки при

$$
\mathrm{k}=0,3
$$

\begin{tabular}{|l|l|l|l|l|l|}
\hline $\mathbf{J}_{\mathbf{H}^{\prime}} \mathbf{K} \boldsymbol{\Gamma} \cdot \mathbf{M}^{2}$ & $\mathbf{6}$ & $\mathbf{9}$ & $\mathbf{1 5}$ & $\mathbf{2 0}$ & $\mathbf{3 0}$ \\
\hline$\sigma, \%$ & 30 & 31 & 35 & 37 & 42 \\
\hline
\end{tabular}

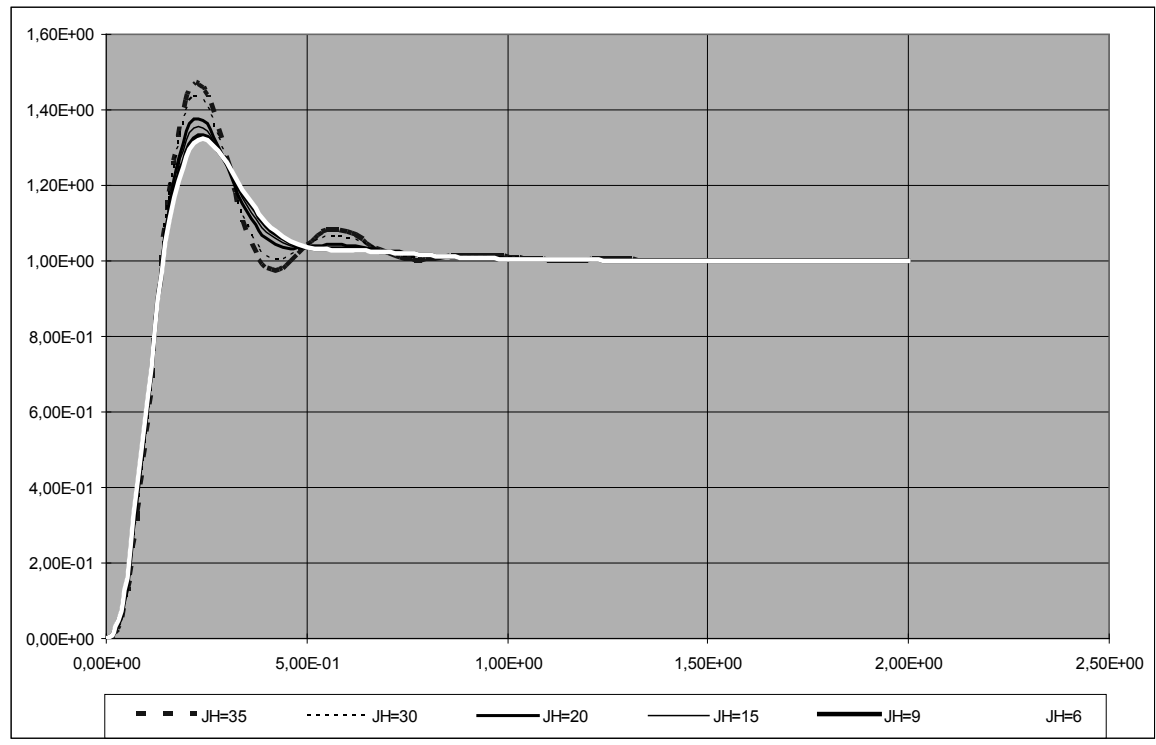

Рис. 8. Переходной процесс при различных значениях момента инерции нагрузки в задаче управления угловым ускорением $(\mathrm{k}=10)$

с перерегулированием в эталонной модели (рис. 6) см. табл. 4.:

При достаточно малом значении $\mathrm{k}=0,3$ система также обладает слабой чувствительностью к изменению параметров нагрузки (таблица 5).

Рассчитаем интегральную квадратичную оценку при различных значениях параметра $\mathrm{k}$ :

$$
\mathrm{I}_{0}=\int_{0}^{3 \mathrm{t}_{\Pi}}\left[\alpha_{\mathrm{M}}(\mathrm{t})-\alpha(\mathrm{t})\right]^{2} \mathrm{dt}
$$

передаточной функции замкнутой системы при различных значениях k:

$$
\lim _{k \rightarrow \infty} p_{\zeta}(k) \rightarrow p_{v}^{*}
$$

могут быть также сведены в таблицу, которая иллюстрирует процесс приближения доминирующих полюсов $\mathrm{p}_{\zeta}(\mathrm{k})$ системы к полюсам $\mathrm{p}_{v}^{*}$ модели. Рассчитаем ЛАЧХ разомкнутой системы: $\mathrm{L}(\omega, \mathrm{k})=20 \lg |\mathrm{W}(\mathrm{j} \omega, \mathrm{k})|(36)$

Моделирование системы с передаточной функцией разомкнутой системы осуществим с помощью схемы с положительной обратной связью (37):

$$
\mathrm{W}(\mathrm{p}, \mathrm{k})=\frac{\mathrm{K}(\mathrm{p}, \mathrm{k})}{1-\mathrm{K}(\mathrm{p}, \mathrm{k})} .
$$

ЛАЧХ и ЛФЧХ для разомкнутой системы в задаче управления угловым ускорением приводятся на рисунке 7. Графические построения логарифмических частотных характеристик выполнены в среде Microsoft Excel (MSExcel).

\section{5. А^горитм управ^ения угловым положением с обратной связью по скорости, исслеАование $\triangle$ инамики системы}

\section{1. Требования к системе управления, эталонная модель}

Повысим требования к синтезируемой системе по величине перерегулирования по сравнению с эталонной моделью рассмотренной в п. 4.1. Длительность переходного процесса: $t_{\Pi}=0,6$ с.Величина перерегулирования: $\sigma \approx 17 \%<20 \%$. Динамическая точность $\varepsilon=0,3 \cdot 10^{-3}$ рад. ЛАФЧХ разомкнутой эталонной модели представлены на рисунке 9. Передаточная функция разомкнутой эталонной модели отвечающей вышеуказанным требованиям: 
Таблица 6. Полюсы передаточной функции замкнутой эталонной модели

\begin{tabular}{|l|l|l|}
\hline $\mathbf{p}_{1}$ & $\mathbf{p}_{\mathbf{2}}$ & $\mathbf{p}_{\mathbf{3}}$ \\
\hline$-33,727$ & $-11,708$ & $-5,065$ \\
\hline
\end{tabular}

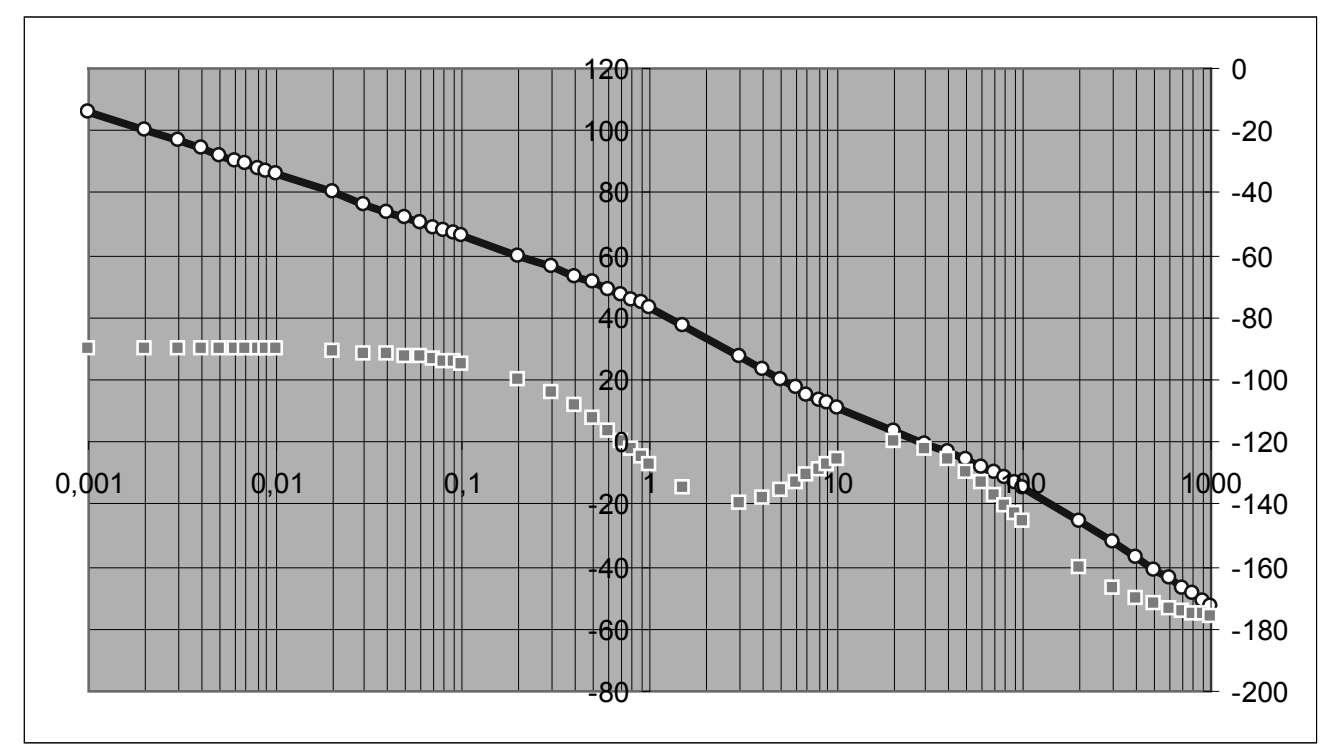

Рис. 9. ЛАФЧХ разомкнутой эталонной модели в задаче управления угловой скоростью
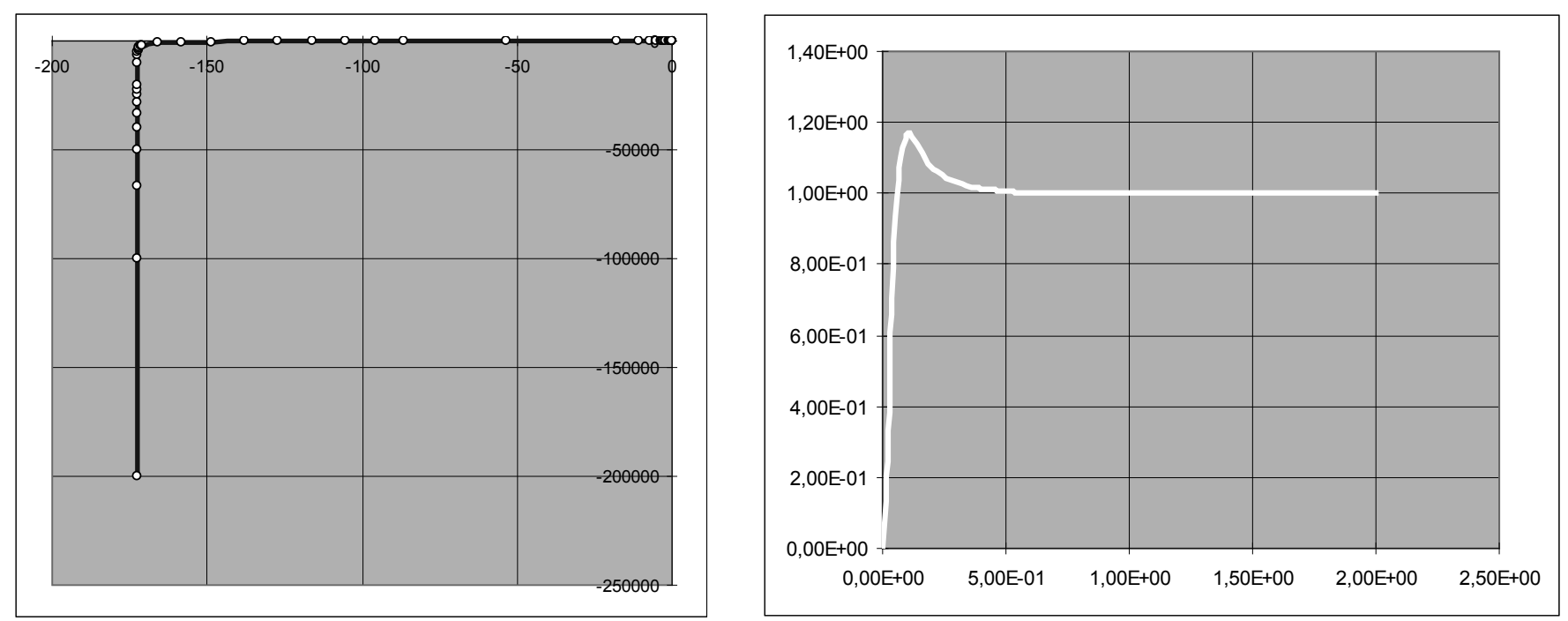

Рис. 10. Переходной процесс и фазовая траектория модели

$$
\mathrm{W}_{\mathrm{M}}=80(0,3 \mathrm{p}+1) / \mathrm{p}(2 \mathrm{p}+1)(0,02 \mathrm{p}+1) .
$$

Переходной процесс и фазовая траектория (годограф) замкнутой эталонной модели с передаточной функцией $\mathrm{K}_{\mathrm{M}}$ приведен на рис. 10.

$$
\begin{aligned}
& \mathrm{K}_{\mathrm{M}}=\frac{80(0,3 \mathrm{p}+1) / \mathrm{p}(2 \mathrm{p}+1)(0,02 \mathrm{p}+1)}{=}= \\
& =\frac{24 \mathrm{p}+80}{0,04 \mathrm{p}^{3}+2,02 \mathrm{p}^{2}+25 \mathrm{p}+80}=\frac{600 \mathrm{p}+2000}{\mathrm{p}^{3}+50,5 \mathrm{p}^{2}+625 \mathrm{p}+2000}
\end{aligned}
$$




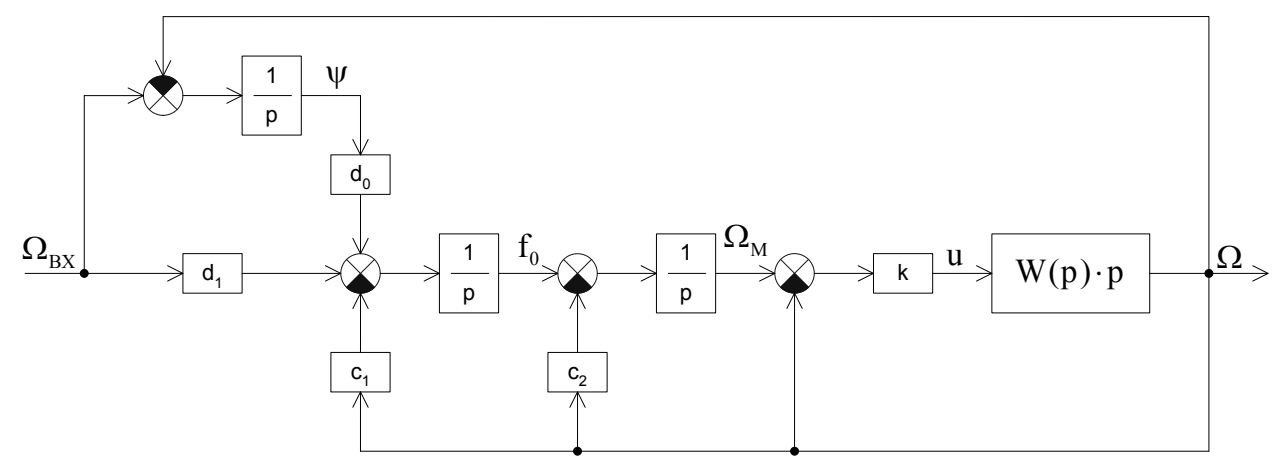

Рис. 11. Синтезированный алгоритм управления угловой скоростью

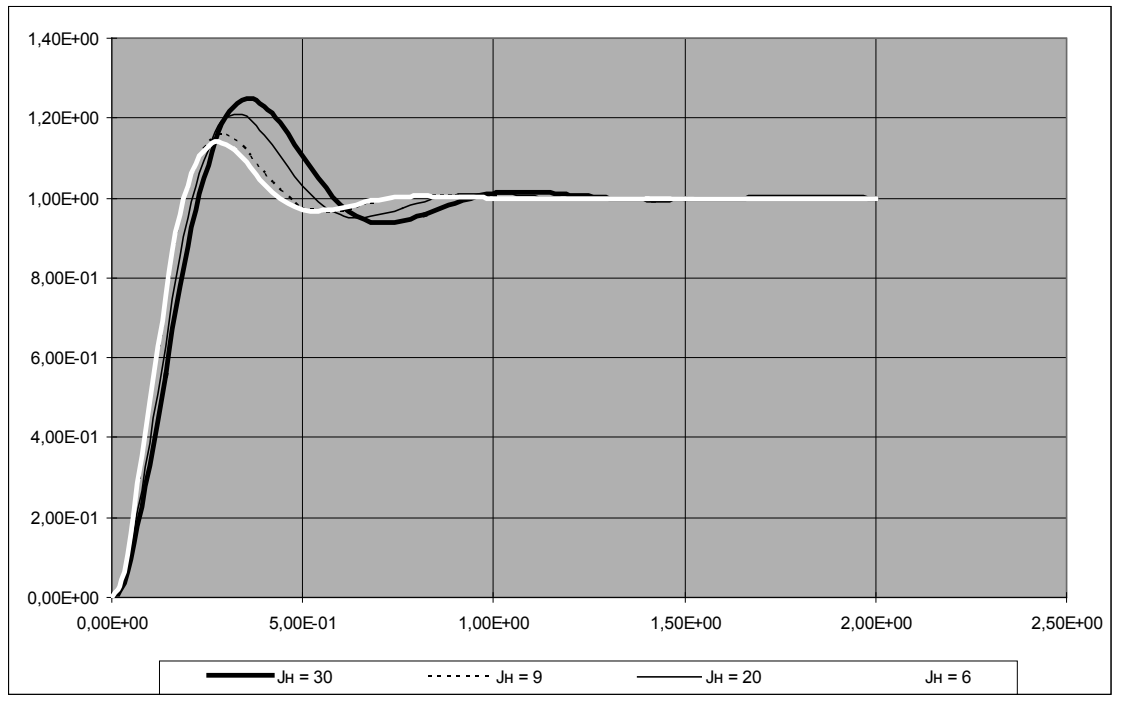

Рис. 12. Изменение скорости на выходе системы рис. 11

Полюсы полученной передаточной функции приведены в таблице 6.

\section{Введем обозначения:}

$\beta_{1}=600, \beta_{0}=2000, \gamma_{2}=50,5, \gamma_{1}=625, \gamma_{0}=2000$

Передаточную функцию замкнутой эталонной модели (ЭМ) в виде:

$$
\mathrm{K}_{\mathrm{M}}(\mathrm{p})=\frac{\beta_{1} \mathrm{p}+\beta_{0}}{\mathrm{p}^{3}+\gamma_{2} \mathrm{p}^{2}+\gamma_{1} \mathrm{p}+\gamma_{0}}=\frac{\alpha_{\mathrm{M}}}{\alpha_{\mathrm{BX}}} .
$$

Требуется, чтобы синтезируемая система повторила поведение ЭМ:

\section{2. Синтез алгоритма управления}

Как ранее указывалось, особенность алгоритма управления (26) состоит в том, что для вычисления управляющей функции необходимо иметь информацию об ускорении $\ddot{\alpha}$. Однако уравнения алгоритма можно преобразовать к такой форме, что для их аппаратной реализации не потребуется информация о второй производной управляемой переменной ӓ. Действительно, дифференциальный закон управления допускает понижение порядка:

$$
\mathrm{u}=\mathrm{k}\left(\dot{\alpha}_{\mathrm{M}}-\dot{\alpha}\right) \text {. }
$$

Дифференциальное уравнение замкнутой эталонной модели:

$$
\dddot{\alpha}_{M}+\gamma_{2} \ddot{\alpha}_{M}+\gamma_{1} \dot{\alpha}_{M}+\gamma_{0} \alpha_{M}=\beta_{1} \dot{\alpha}_{B X}+\beta_{0} \alpha_{B X} \text {. }
$$

Выразим первую производную выходной координаты:

$$
\begin{aligned}
& \dddot{\alpha}_{M}=\beta_{1} \dot{\alpha}_{B X}+\beta_{0} \alpha_{B X}-\gamma_{2} \ddot{\alpha}_{M}-\gamma_{1} \dot{\alpha}_{M}-\gamma_{0} \alpha_{M}, \\
& \ddot{\alpha}_{M}=\beta_{1} \alpha_{B X}+\gamma_{0} \int_{0}^{t}\left(\alpha_{B X}-\alpha_{M}\right) d t-\gamma_{2} \dot{\alpha}_{M}-\gamma_{1} \alpha_{M},
\end{aligned}
$$




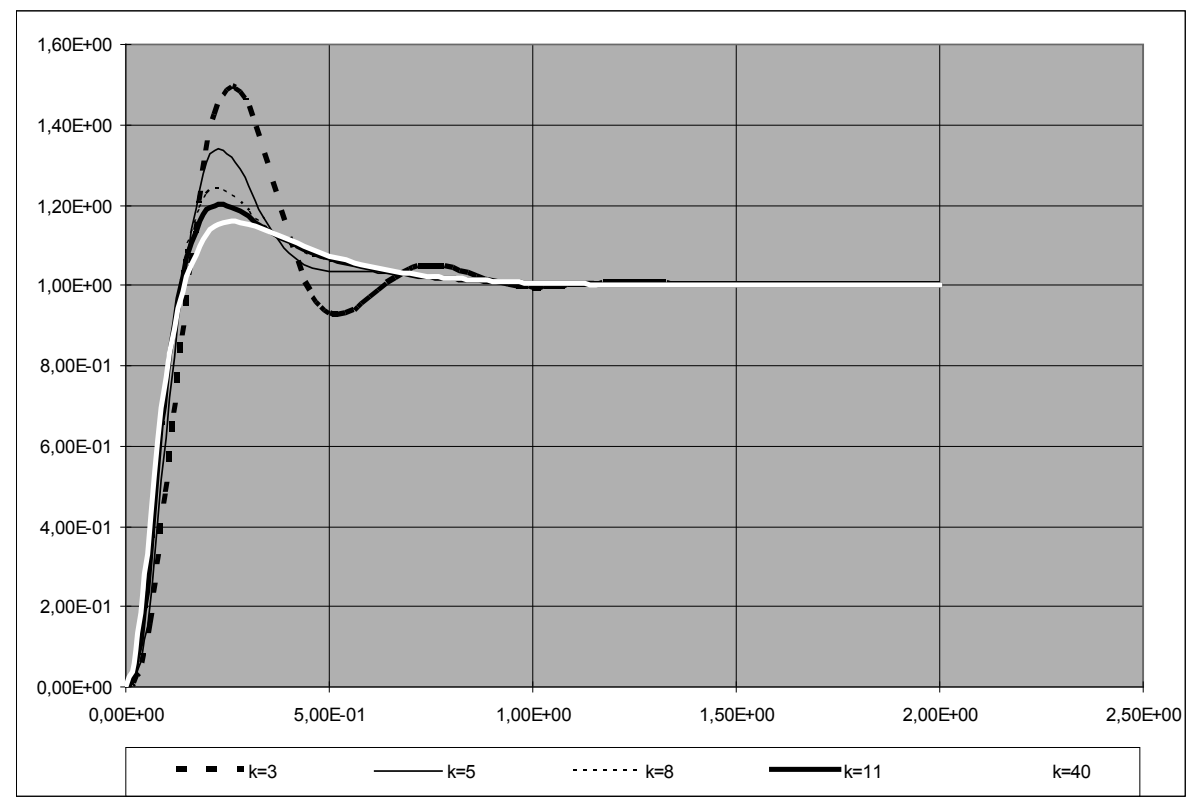

Рис. 13. САУ управления угловой скоростью (настройка k)

$\psi_{\mathrm{M}}=\gamma_{0} \int_{0}^{\mathrm{t}}\left(\alpha_{\mathrm{BX}}-\alpha_{\mathrm{M}}\right) \mathrm{dt}$

$\dot{\alpha}_{M}=\int_{0}^{t}\left(\psi_{M}+\beta_{1} \alpha_{B X}-\gamma_{1} \alpha_{M}\right) d t-\gamma_{2} \alpha_{M}$.

Однако вычислять управляющую функцию необходимо с учетом состояния управляемого объекта $\left(\alpha_{M}=\alpha, \dot{\alpha}_{M}=\dot{\alpha}\right)$.

Тогда уравнения (47), с учетом выражения (1) для объекта управления, полностью определяют алгоритм управления с обратной связью по скорости.

$$
\begin{aligned}
& \dot{\alpha}_{\mathrm{M}}=\int_{0}^{\mathrm{t}}\left(\psi_{\mathrm{M}}+\beta_{1} \alpha_{\mathrm{BX}}-\gamma_{1} \alpha\right) \mathrm{dt}-\gamma_{2} \alpha \\
& \psi=\gamma_{0} \int_{0}^{\mathrm{t}}\left(\alpha_{\mathrm{BX}}-\alpha\right) \mathrm{dt}, \\
& \mathrm{u}=\mathrm{k}\left(\dot{\alpha}_{\mathrm{M}}-\dot{\alpha}\right)
\end{aligned}
$$

Структурная схема замкнутой системы для управления угловым положением с обратной связью по скорости приведена на рисунке 11.

\section{3. Исследование динамики системы}

Прежде всего, подберем такой коэффициент усиления, при котором переходной процесс асимптотически приближается к эталонному процессу.

На рисунке 13 приводится вид переходного процесса при $\mathrm{k}=\mathrm{k}^{*}=40$, из которого видно, что при данном значении коэффициента усиления, переходной процесс в синтезированной системе весьма близок к переходному процессу в эталонной модели (см. рис. 10).

Параметр настройки h. Передаточная функция замкнутой эталонной модели имеет вид: $\mathrm{K}_{\mathrm{M}}^{\Omega}=\frac{\mathrm{d}(\mathrm{p})}{\mathrm{c}(\mathrm{p})}=\frac{\Omega^{*}}{\Omega_{\mathrm{BX}}}=\frac{\mathrm{d}_{1} \mathrm{p}+\mathrm{d}_{0}}{\mathrm{p}^{3}+\mathrm{c}_{2} \mathrm{p}^{2}+\mathrm{c}_{1} \mathrm{p}+\mathrm{c}_{0}}$.

\section{4. Обоснование устойчивости системы по критерию Гурвица}

Для иллюстрации не требуется рассмотрения объекта управления (ОУ) высокого порядка. Рассмотрим иллюстративно передаточную функция ОУ 3-го порядка:

$$
\mathrm{W}_{0}^{\Omega}(\mathrm{p})=\frac{\mathrm{b}_{0}}{\mathrm{p}^{3}+\mathrm{a}_{3}^{\prime} \mathrm{p}^{2}+\mathrm{a}_{2}^{\prime} \mathrm{p}+\mathrm{a}_{1}^{\prime}}=\frac{\mathrm{b}_{0}}{\mathrm{~A}(\mathrm{p})}=\frac{\Omega}{\mathrm{u}}
$$

Необходимым условием критерия Гурвица является положительность коэффициентов характеристического многочлена. Для анализа устойчивости необходимо выразить передаточную функцию замкнутой системы:

$$
\mathrm{K}^{\Omega}(\mathrm{p})=?=\frac{\Omega}{\Omega_{\mathrm{BX}}} .
$$

С учетом закона управления $\mathrm{u}=\mathrm{kp}\left(\Omega^{*}-\Omega\right)-\frac{\mathrm{h}}{\mathrm{p}} \mathrm{u}$, записанном в виде

$$
\mathrm{u}\left[1+\frac{\mathrm{h}}{\mathrm{p}}\right]=\mathrm{kp}\left(\Omega^{*}-\Omega\right)
$$

проведем ряд преобразований: 
Таблица 7. Полюсы передаточной функции замкнутой эталонной модели

\begin{tabular}{|l|l|l|}
\hline $\mathbf{p}_{1}$ & $\mathbf{p}_{2}$ & $\mathbf{p}_{3}$ \\
\hline$-33,727$ & $-11,708$ & $-5,065$ \\
\hline
\end{tabular}

$$
\begin{aligned}
& \mathrm{u}\left[1+\frac{\mathrm{h}}{\mathrm{p}}\right]=\mathrm{kp}\left(\frac{\mathrm{d}(\mathrm{p})}{\mathrm{c}(\mathrm{p})} \Omega_{\mathrm{BX}}-\Omega\right) \\
& \frac{\mathrm{A}(\mathrm{p})}{\mathrm{b}_{0}}\left[1+\frac{\mathrm{h}}{\mathrm{p}}\right] \Omega=\mathrm{kp}\left(\frac{\mathrm{d}(\mathrm{p})}{\mathrm{c}(\mathrm{p})} \Omega_{\mathrm{BX}}-\Omega\right) \\
& {\left[\frac{\mathrm{A}(\mathrm{p})}{\mathrm{b}_{0}}\left(\frac{\mathrm{p}+\mathrm{h}}{\mathrm{p}}\right)+\mathrm{kp}\right] \Omega=\mathrm{kp} \frac{\mathrm{d}(\mathrm{p})}{\mathrm{c}(\mathrm{p})} \Omega_{\mathrm{BX}},} \\
& \frac{\mathrm{kp} \frac{\mathrm{d}(\mathrm{p})}{\mathrm{c}(\mathrm{p})}}{\frac{\mathrm{A}(\mathrm{p})}{\mathrm{b}_{0}} \frac{(\mathrm{p}+\mathrm{h})}{\mathrm{p}}+\mathrm{kp}}=\frac{\Omega}{\Omega_{\mathrm{BX}}} .
\end{aligned}
$$

Передаточная функция замкнутой системы разрешенная относительно $\mathrm{k}$ и $\mathrm{h}$

$$
\frac{\Omega}{\Omega_{B X}}=\frac{b_{0} k p^{2} d(p)}{c(p) A(p)(p+h)+k p^{2} b_{0} c(p)} .
$$

Многочлен с(p) имеет 3 порядок. Многочлен A(p) имеет 3 порядок. Произведение многочленов $c(p) A(p)(p+h)$ имеет 7 порядок $(3+3+1=7)$. Произведение $\mathrm{kp}^{2} \mathrm{~b}_{0} \mathrm{c}(\mathrm{p})$ имеет 5 порядок $(3+2=5)$. Коэффициенты при старших степенях р не зависят k. Однако за счет изменения параметра $h$ можно скомпенсировать отрицательные коэффициенты при старших степенях р и сделать систему устойчивой. Таким образом, в оконечной реализации синтезированных систем присутствует две (2-е) ручки настройки $\mathrm{h}$ и k.

\section{6. Алгоритм управ^ения угловым по^ожением с обратной связьЮ \\ по скорости, исслеАование $\triangle$ инамикИ системы}

\section{1. Требования к системе управления, эталонная модель}

Повысим требования к синтезируемой системе по величине перерегулирования по сравнению с эталонной моделью рассмотренной в п. 4. Длительность переходного процесса: $\mathrm{t}_{\Pi}=0,6$ с. Величина перерегулирования: $\sigma \approx 17 \%<20 \%$. Динамическая точность $\varepsilon=0,3 \cdot 10^{-3}$ рад. Передаточная функция разомкнутой эталонной модели отвечающей вышеуказанным требованиям:

$$
\mathrm{W}_{\mathrm{M}}=80(0,3 \mathrm{p}+1) / \mathrm{p}(2 \mathrm{p}+1)(0,02 \mathrm{p}+1) .
$$

Передаточная функция замкнутой эталонной модели:

$$
\begin{aligned}
& \mathrm{K}_{\mathrm{M}}=\frac{80(0,3 \mathrm{p}+1) / \mathrm{p}(2 \mathrm{p}+1)(0,02 \mathrm{p}+1)}{1+80(0,3 \mathrm{p}+1) / \mathrm{p}(2 \mathrm{p}+1)(0,02 \mathrm{p}+1)}= \\
& =\frac{24 \mathrm{p}+80}{0,04 \mathrm{p}^{3}+2,02 \mathrm{p}^{2}+25 \mathrm{p}+80}=\frac{600 \mathrm{p}+2000}{\mathrm{p}^{3}+50,5 \mathrm{p}^{2}+625 \mathrm{p}+2000}
\end{aligned}
$$

Полюсы полученной передаточной функции приведены в таблице 2.7.

\section{Введем обозначения:}

$$
\beta_{1}=600, \beta_{0}=2000, \gamma_{2}=50,5, \gamma_{1}=625, \gamma_{0}=2000 \text {. }
$$

Передаточную функцию замкнутой эталонной модели в виде:

$$
K_{M}(p)=\frac{\beta_{1} p+\beta_{0}}{p^{3}+\gamma_{2} p^{2}+\gamma_{1} p+\gamma_{0}}=\frac{\alpha_{M}}{\alpha_{\tilde{Q}}}
$$

Переходной процесс в эталонной модели ЛАФЧХ и годограф разомкнутой эталонной модели приводить не будем, сразу посмотрим сигнал на выходе синтезированной системы.

\section{2. Синтез алгоритма управления}

Особенность алгоритма управления угловым ускорением состоит в том, что для вычисления управляющей функции необходимо иметь информацию об ускорении $\ddot{\alpha}$. Однако уравнения алгоритма можно преобразовать к такой форме, что для их аппаратной реализации не потребуется информация о второй производной управляемой переменной $\ddot{\alpha}$. Действительно, дифференциальный закон управления допускает понижение порядка:

$$
\mathrm{u}=\mathrm{k}\left(\dot{\alpha}_{\mathrm{M}}-\dot{\alpha}\right)
$$

Дифференциальное уравнение замкнутой эталонной модели:

$$
\dddot{\alpha}_{M}+\gamma_{2} \ddot{\alpha}_{M}+\gamma_{1} \dot{\alpha}_{M}+\gamma_{0} \alpha_{M}=\beta_{1} \dot{\alpha}_{B X}+\beta_{0} \alpha_{B X}
$$

Выразим первую производную выходной координаты:

$$
\dddot{\alpha}_{M}=\beta_{1} \dot{\alpha}_{B X}+\beta_{0} \alpha_{B X}-\gamma_{2} \ddot{\alpha}_{M}-\gamma_{1} \dot{\alpha}_{M}-\gamma_{0} \alpha_{M},
$$




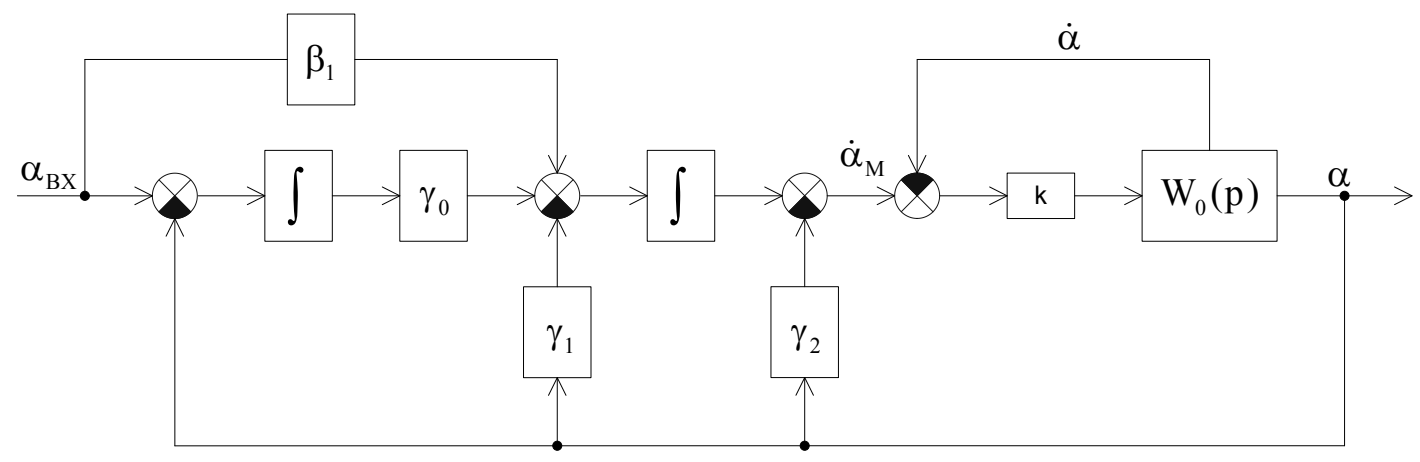

Рис. 14. Синтезированный алгоритм управления угловым положением

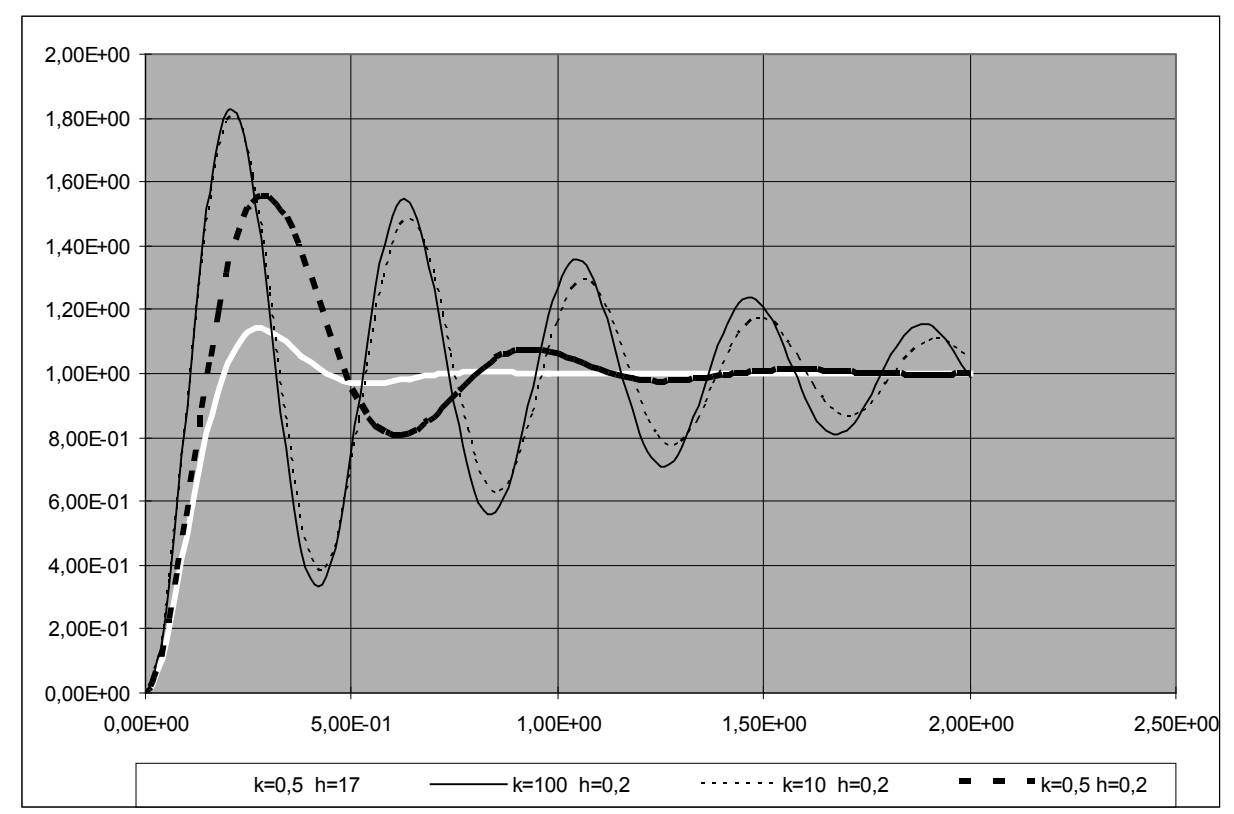

Рис. 15. Угол поворота на выходе в синтезированной системы

$\ddot{\alpha}_{M}=\beta_{1} \alpha_{B X}+\gamma_{0} \int_{0}^{t}\left(\alpha_{B X}-\alpha_{M}\right) d t-\gamma_{2} \dot{\alpha}_{M}-\gamma_{1} \alpha_{M}$,

$\psi_{\mathrm{M}}=\gamma_{0} \int_{0}^{\mathrm{t}}\left(\alpha_{\mathrm{BX}}-\alpha_{\mathrm{M}}\right) \mathrm{dt}$,

$\dot{\alpha}_{M}=\int_{0}^{t}\left(\psi_{M}+\beta_{1} \alpha_{B X}-\gamma_{1} \alpha_{M}\right) d t-\gamma_{2} \alpha_{M}$.

Однако, вычислять управляющую функцию необходимо с учетом состояния управляемого объекта $\left(\alpha_{M}=\alpha, \dot{\alpha}_{M}=\dot{\alpha}\right)$.

Тогда уравнения (64)-(66) с учетом выражения (1) для объекта управления, полностью определяют алгоритм управления с обратной связью по скорости.

$$
\dot{\alpha}_{M}=\int_{0}^{t}\left(\psi_{M}+\beta_{1} \alpha_{B X}-\gamma_{1} \alpha\right) \mathrm{dt}-\gamma_{2} \alpha
$$

$$
\begin{aligned}
& \psi=\gamma_{0} \int_{0}^{\mathrm{t}}\left(\alpha_{\mathrm{BX}}-\alpha\right) \mathrm{dt}, \\
& \mathrm{u}=\mathrm{k}\left(\dot{\alpha}_{\mathrm{M}}-\dot{\alpha}\right) .
\end{aligned}
$$

Структурная схема замкнутой системы для управления угловым положением с обратной связью по скорости приведена на рисунке 14.

\section{3. Исследование динамики замкнутой системы}

На рис. 15 приведен вид переходного процесса для фиксированном значения момента инерции нагрузки $\left(\mathrm{J}_{\mathrm{H}}=6\right)$.

При различных значениях параметров регулирования, в частности при $\mathrm{k}=0,5$ и $\mathrm{h}=0,5$ достигается вид пе- 


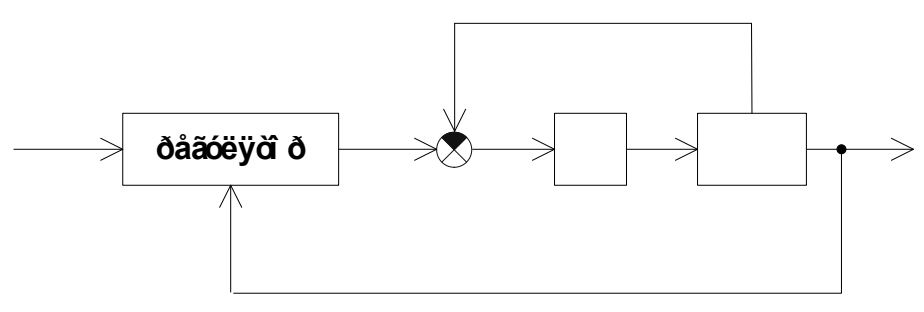

Рис. 16. Аналоговый прототип, полученный в результате синтеза

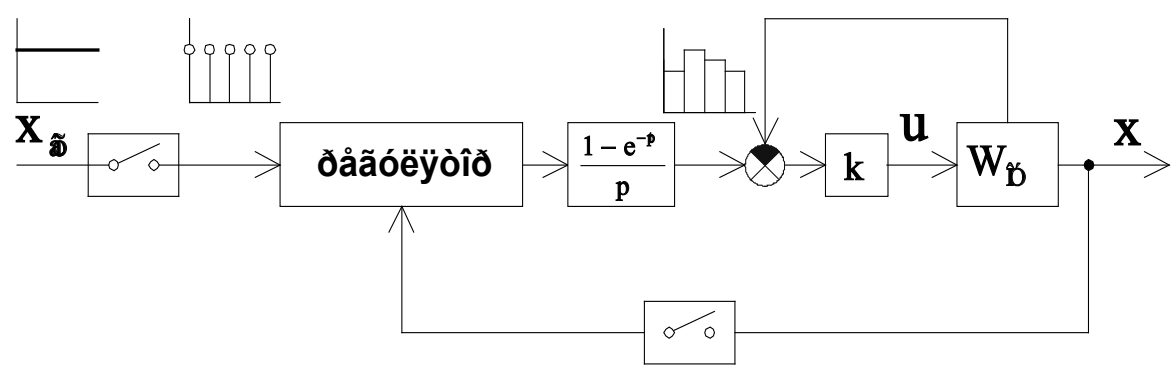

Рис. 17. Цифрой адаптированный алгоритм на основе прототипа

реходного процесса отвечающий требованиям предъявляемым к синтезируемой системе.

Сделаем важный вывод - хороший результат не всегда достигается при непомерно большом коэффициенте усиления прямого тракта. Пропорцию настройки следует выбирать, в данном случае характеристика системы близка к эталонной модели при $\mathrm{k}<1$.

\section{7. Шифровые алгоритмы управ^ения}

\section{1. Методика построения цифровых алгоритмов управления}

Выделим основные этапы построения цифровых алгоритмов, которые далее используем в данной дипломной работе: (1) для построения цифровой системы, будем проводить синтез соответствующего аналогового алгоритма, после чего при помощи введения дискретных звеньев преобразуем полученную аналоговую систему в цифровую; (2) контур управляющей функции оставим непрерывным, также как и обратную связь по старшей производной, входящей в уравнение закона управления; (3).измерение переменных состояния производятся цифровыми измерителями; (4) для преобразования дискретного сигнала в непрерывный ступенчатый сигнал воспользуемся экстраполятором нулевого порядка.

Пусть в процессе проведения синтеза аналогового алгоритма управления получена система со структурной схемой, изображенной на рисунке 16. Здесь "u" -управляющая функция.
Структурная схема соответствующей цифровой системы примет вид представленный на рисунке 17.

Заметим, что в состав звеньев синтезированных аналоговых алгоритмов управления угловым положением упругой двухмассовой системы в входят интегрирующие и усилительные звенья.

Напомним, что уравнения алгоритма управления угловым положением с обратной связью по ускорению выглядели следующим образом:

$$
\begin{aligned}
& \ddot{\alpha}_{\mathrm{M}}=\mathrm{f}_{0}-\gamma_{2} \alpha-\gamma_{3} \dot{\alpha}, \\
& \mathrm{u}=\mathrm{k}\left(\ddot{\alpha}_{\mathrm{M}}-\ddot{\alpha}\right), \\
& \text { где, } \mathrm{f}_{0}=\int_{0}^{\mathrm{t}}\left[\gamma_{0} \psi+\beta_{1} \alpha_{\mathrm{BX}}-\gamma_{1} \alpha\right] \mathrm{dt}, \\
& \text { a } \psi=\int_{0}^{\mathrm{t}}\left(\alpha_{\mathrm{BX}}-\alpha\right) \mathrm{dt} .
\end{aligned}
$$

При построении дискретной системы контур ускорения оставим аналоговым, тогда:

$$
\begin{aligned}
& \ddot{\alpha}_{\mathrm{M}}(v \mathrm{~T})=\mathrm{f}_{0}(v \mathrm{~T})-\gamma_{2} \alpha(v \mathrm{~T})-\gamma_{3} \dot{\alpha}(v \mathrm{~T}) . \\
& \mathrm{f}_{0}(\mathrm{t})=\mathrm{f}_{0}(v \mathrm{~T})=? \quad \psi(v \mathrm{~T})=?
\end{aligned}
$$

Разобьем интеграл $\mathrm{f}_{0}$ на два интеграла:

$$
\mathrm{f}_{0}=\int_{0}^{\mathrm{t}} \varphi(\mathrm{t}) \mathrm{dt}=\int_{0}^{v \mathrm{~T}} \varphi(\mathrm{t}) \mathrm{dt}=\int_{0}^{(v-1) \mathrm{T}} \varphi(\mathrm{t}) \mathrm{dt}+\int_{(v-1) \mathrm{T}}^{v \mathrm{~T}} \varphi(\mathrm{t}) \mathrm{dt},
$$



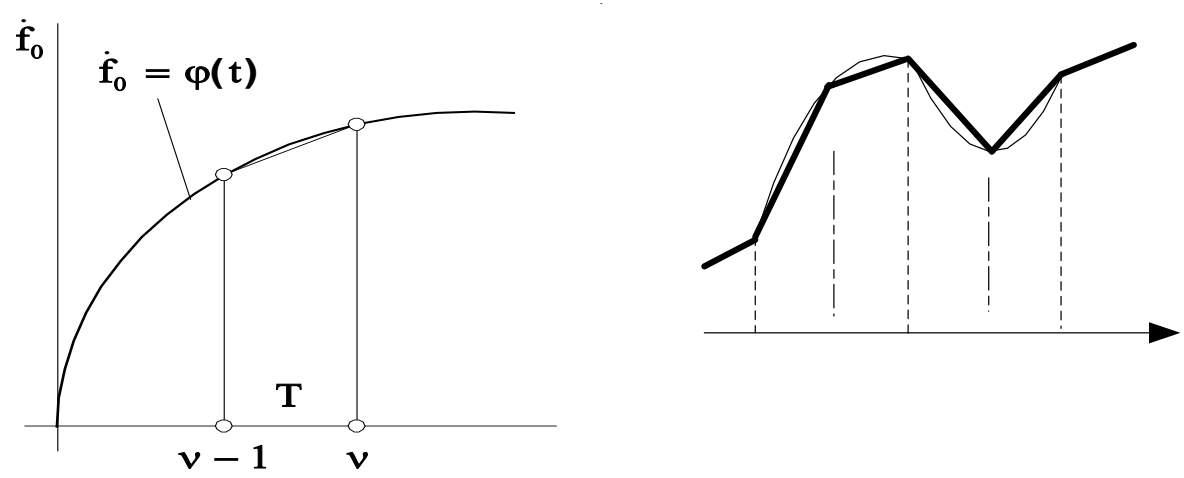

Рис. 18. Замена кривой ломанной с повышением частоты в 2 раза

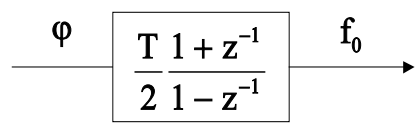

Рис. 19. Квадратура интегратора

$$
\mathrm{f}_{0}=\mathrm{I}(v-1)+\int_{(v-1) \mathrm{T}}^{v \mathrm{~T}} \varphi(\mathrm{t}) \mathrm{dt}=\mathrm{I}(\mathrm{v})
$$

где $\mathrm{T}$ - период дискретности, $\psi(\mathrm{t})=\dot{\mathrm{f}}_{0}(\mathrm{t})$,

Заметим, что $\dot{\psi}(\mathrm{t})=\left(\alpha_{\mathrm{Bx}}-\alpha\right)=\varepsilon(\mathrm{t}) .(73)$

Для того, чтобы повысить частоту квантования в два раза следует решать задачу Коши с уточнением дифференциалов левой и правой границы трапеции квадратуры. Действительно для правой границы заранее не известно, но предопределено наиболее вероятное значение, а вот промежуточная точка определена полностью и является закономерной позицией при условии константности параметров системы. Тогда, можно по четным и нечетным узлам, а следовательно и для любого узла получить точный прогноз изменения знака выхода, а затем и полностью уточнить амплитуду с учетом знака и динамики параметров. Уравнения для изменения знака будут дополнительными для задачи Коши.

Для первого шага можно получить довольно близкий дифференциал и амплитуду, а вот на втором шаге гарантировать точное вычисление через шаг уже нельзя. Анализируя динамику числа степеней свободы системы можно определить с какой, неопределенностью ведется точный прогноз, да могут быть итерации где амплитуда на следующем промежуточном шаге не имеет полной матовости, но можно дописать дополнительные уравнения, чтобы использовать их в процессе синтеза дискретного алгоритма. Можно утверждать, что на первой итерации будет, по крайней мере, правильно выбран знак, а на следующей четной - верная амплитуда, правда при условии калибровки выхода, поэтому имеет смысл повысить частоту именно в два (2) раза, что приводит к алгоритмам управления знаком выхода для поддержки режимов резкого реверса. Однако, можно попытаться совместить статический режим со сменой знака сигнала и последующей отработкой задающего воздействия, для пульта с коробкой переключений.

Указанный интеграл $\mathrm{f}_{0^{\prime}}$ как показано, обладает рекурсивным свойством. Для его вычисления воспользуемся 18.

Квадратурные преобразования требуют отдельной статьи, поэтому рассмотрим сначала простую квадратуру с сохранением частоты в качестве иллюстрации, не вдаваясь в проблему повышения частоты с учетом мониторинга дифференциала координат непрерывного объекта. Составляющая

$$
\int_{(v-1) \mathrm{T}}^{v \mathrm{~T}} \varphi(\mathrm{t}) \mathrm{dt}
$$

может быть вычислена как площадь трапеции:

$$
\begin{aligned}
& \int_{(v-1) \mathrm{T}}^{v \mathrm{~T}} \varphi(\mathrm{t}) \mathrm{dt}=\frac{\mathrm{T}}{2}[\varphi(v-1)+\varphi(v)] \\
& \mathrm{f}_{0}=\mathrm{I}(v)=\mathrm{I}(v-1)+\frac{\mathrm{T}}{2}[\varphi(v-1)+\varphi(v)] \\
& \mathrm{L}^{*}\{\varphi(v)\}=\varphi^{*}(\mathrm{z}) ; \mathrm{z}=\mathrm{e}^{\mathrm{pT}} \\
& \mathrm{L}^{*}\{\varphi(v-1)\}=\varphi^{*}(\mathrm{z}) \frac{1}{\mathrm{z}}=\varphi^{*}(\mathrm{z}) \cdot \mathrm{z}^{-1} \\
& \mathrm{~L}^{*}\{\mathrm{I}(v)\}=\mathrm{I}^{*}(\mathrm{z}) ; \mathrm{L}^{*} \quad\{\mathrm{I}(v-1)\}=\mathrm{I}^{*}(\mathrm{z}) \cdot \mathrm{z}^{-1} \\
& \mathrm{I}^{*}(\mathrm{z})-\mathrm{I}^{*}(\mathrm{z}) \cdot \mathrm{z}^{-1}=\frac{\mathrm{T}}{2}\left(1+\mathrm{z}^{-1}\right) \varphi^{*}(\mathrm{z})
\end{aligned}
$$




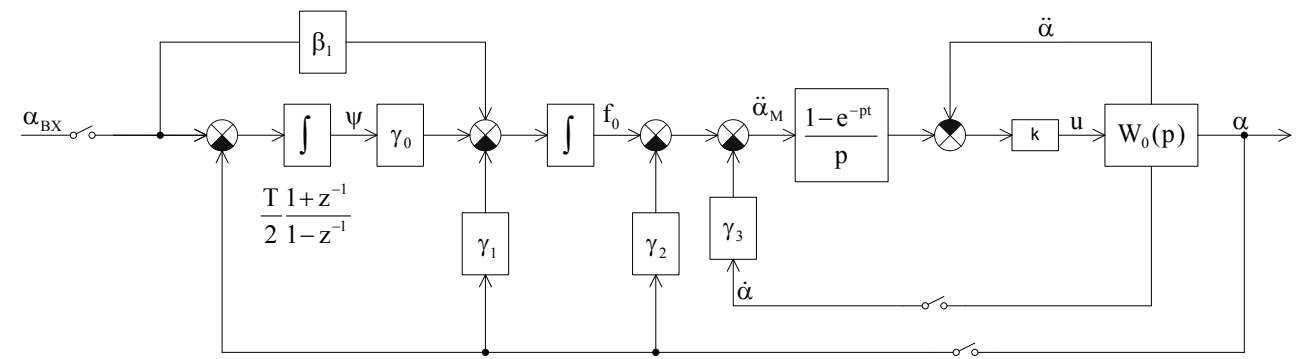

Рис. 20. Дискретный алгоритм управления угловым положением с обратной связью по ускорению

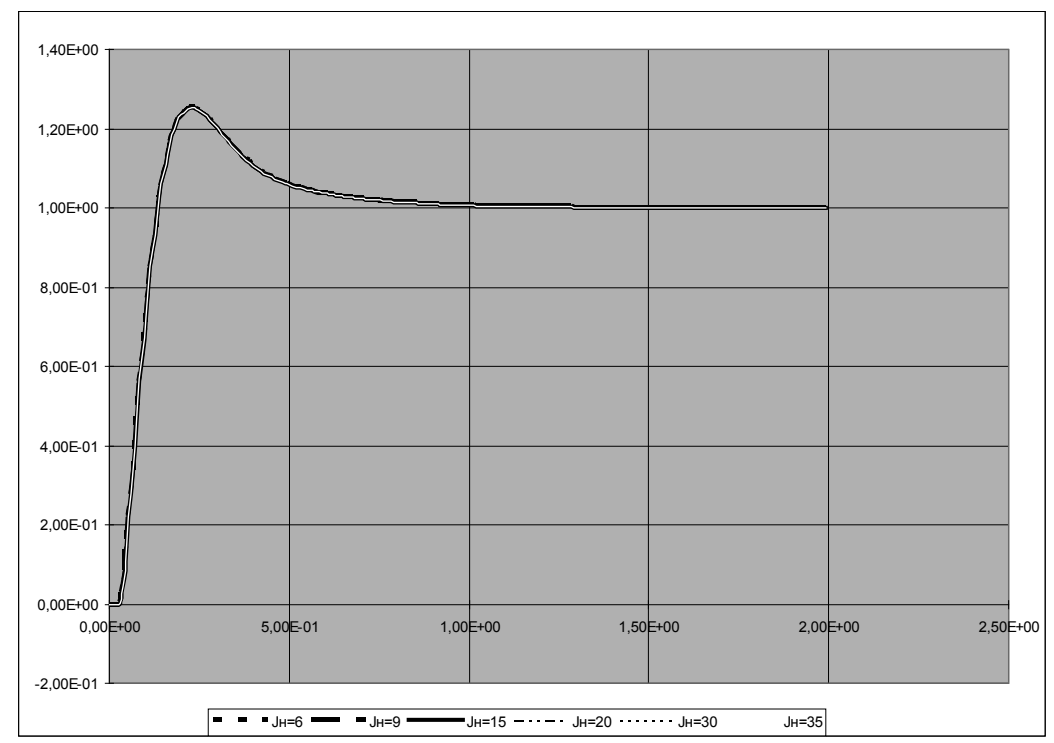

Рис. 21. Переходной процесс в системе с дискретным алгоритмом k=10

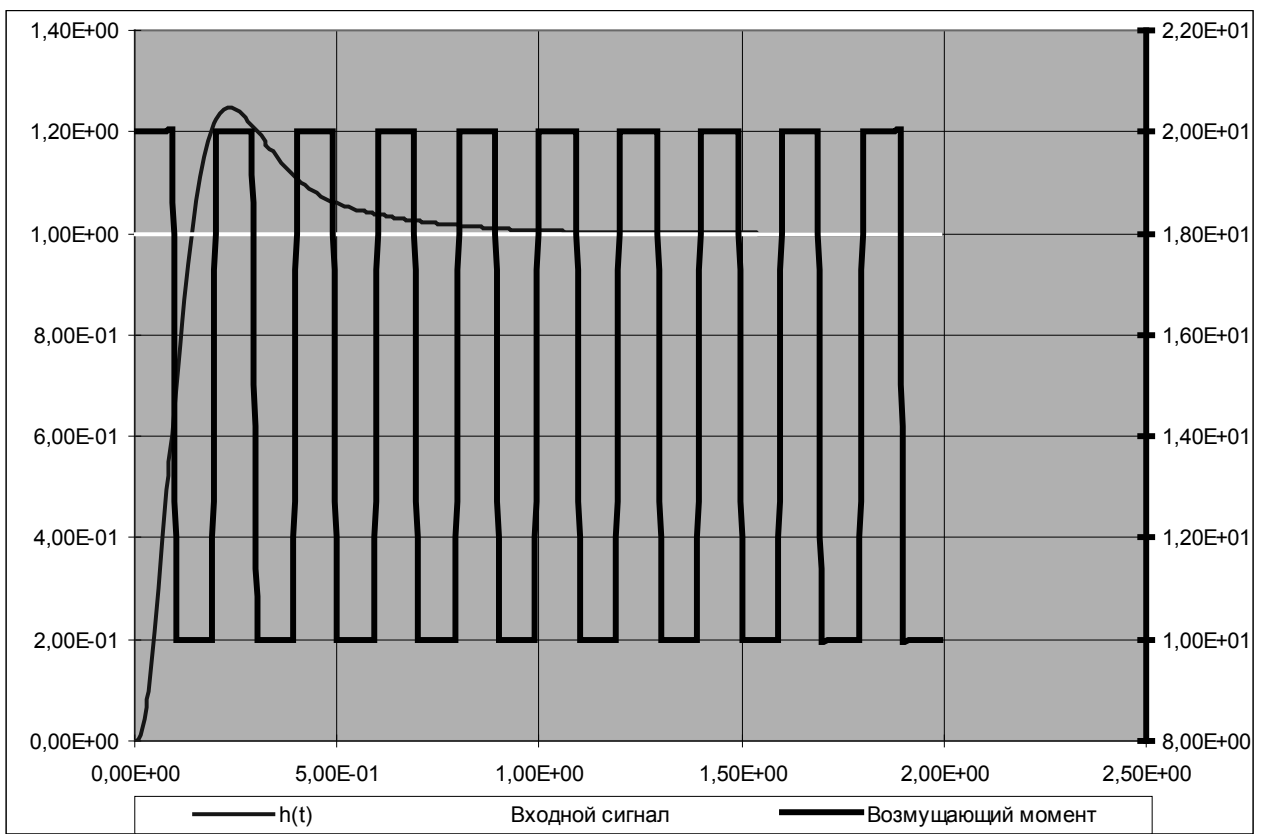

Рис. 22. Возмущение и переходной процесс в системе с дискретным алгоритмом управления. Јн=30 


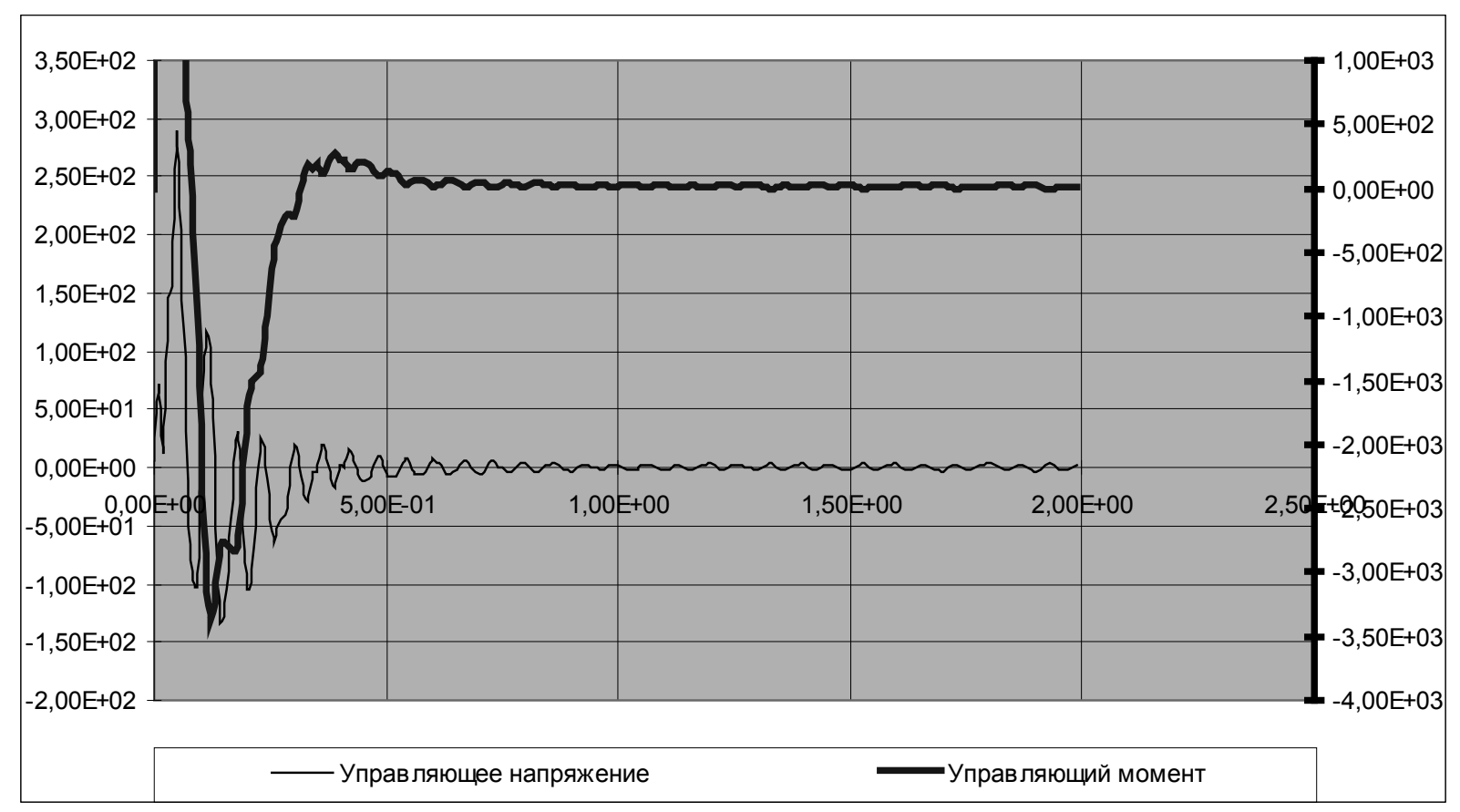

Рис. 23. Управляющий момент и Uупр в системе с дискретным алгоритмом управления (Jн=30) и возмущающим моментом на выходе

$$
\mathrm{f}_{0}^{*}(\mathrm{z})=\mathrm{I}^{*}(\mathrm{z})=\mathrm{I} *(\mathrm{z})=\frac{\mathrm{T}}{2} \frac{1+\mathrm{z}^{-1}}{1-\mathrm{z}^{-1}} \varphi^{*}(\mathrm{z})
$$

Таким образом, схема для моделирования интегратора примет вид изображенный на рисунке 19.

\section{2. Алгоритм управления с обратной связью по угловому ускорению}

Появляется возможность представить аналоговый алгоритм в цифровом виде. Проверить его работу в цифровом виде можно в среде модулирования MatLab Simulink. Модель объекта останется аналоговой, а звенья будут реализованы на базе дискретного преобразования Лапласа с квантованием трактов и обратных связей в цифровом виде, что допустимо в среде Matlab.

Для алгоритмов управления углом поворота и угловой скоростью можно аналогичным образом выполнить преобразование схемы пригодное для реализации в составе микропроцессорных систем.

\section{3. Исследование динамики с возмущающим моментом}

Эквивалентный управляющий момент и управляющее напряжение в системе с возмущением (рисунок 22). приводится на рисунке 23.

\section{Зак^ючение}

В статье рассмотрены методические основы синтеза алгоритмов управления следящих систем высокой динамической точности и малой параметрической чувствительности. Синтез алгоритма управления проводится из условия минимума квадрата энергии ускорения, нормированной по моменту инерции нагрузки, вычисляемой в окрестности траектории движения эталонной модели. Для получения эффективного алгоритма управления необходимо отказаться от достижения абсолютного минимума квадрата энергии, т.е. равенства ее нулю.

По вышеизложенной методике для двухмассовой упругой системы проведен синтез алгоритма управления с малым информационным обеспечением, т.е. для вычисления управляющей функции требуется иметь информацию о положении и скорости. Синтезированные алгоритмы обеспечивают управление угловым положением и угловой скоростью. В процессе исследования динамики замкнутых систем с алгоритмами управления, синтезированными по вышеизложенной методике, установлено, что такие системы обладают слабой параметрической чувствительностью по отношению к естественным возмущениям (изменениям параметров объекта управления) и координационным возмущениям (возмущающим воздействиям). Процедура синтеза оказывается типовой для разного порядка производной 
координаты выхода системы. Для синтеза закона управления объектом более высокого порядка можно пользоваться законом управления, полученным для объекта управления второго порядка, что, в частности, можно объяснить наличием у передаточной функции объекта управления доминирующих полюсов. Это означает, что закон управления - фундаментальное понятие, приме- нимое к различным объектам управления вне зависимости от их порядка и структуры. В результате преобразования непрерывной замкнутой системы в дискретную, свойства параметрической адаптивности к изменению параметров объекта управления сохраняются. Кроме того, дискретная система остается малочувствительной к изменению периода дискретности.

\section{ЛИТЕРАТУРА}

1. Волошиновский К. И. Управление двухмассовой системой с двигателем постоянного тока и рысканьем по выходу. Горный информационно-аналитический бюллетень № 05, 2016 г.- - 17-34 с.

2. Волошиновский К. И. Исследование и испытание приборов и систем. Учеб. пособие М.: МГГУ, 2014. - 120 с.

3. Волошиновский К. И. Аргумент и аргумент с помехой. Горный информационно-аналитический бюллетень № 1, 2017 г., - 66-83 с.

4. Певзнер Л. Д. Теория систем управления. М.: МГГУ, 2002. - 472 с.

5. Певзнер Л. Д. Практикум по теории автоматического управления. Учеб. пособие. М. Высш. шк., 2006. — 590 с.

6. П.Д. Крутько Вариационные методы синтеза систем с цифровыми регуляторами. М. Советское радио 1967,- 438 с

7. Голованов М.А., Иванов М. А. Теория цифровых систем автоматического управления. Часть 1. Учеб. пособ.— М.: Изд-во МГТУ. 1990. — 70 с., ил.

8. Сборник задач по теории автоматического регулирования и управления. Под ред. В. А. Бесекерского. Изд. 5-е,; М.: Наука, 1978, — 512 с.

9. Задачник по теории автоматического управления. Учеб. пособие для вузов Андреев Н. И. и др. /Под ред. А.С. Шаталова 2-е изд., перераб. и доп.М. Энергия, 1979.- 544 с.

10. В.А. Бесекерский. Е. П. Попов. Теория систем автоматического регулирования. М.: Наука, 1966, — 992 с.

11. Копылов И. П. Применение вычислительных машин в инженерно-эконмических расчетах.—М.: Высшая школа, 1980.-256 с.

12. Ключев В. И. Теория электропривода: М.: Энергоатомиздат, 2001-704 С.

13. Вешеневский С. Н. Характеристики двигателей в электроприводе. М.: «Энергия», 1977. - 432 с.

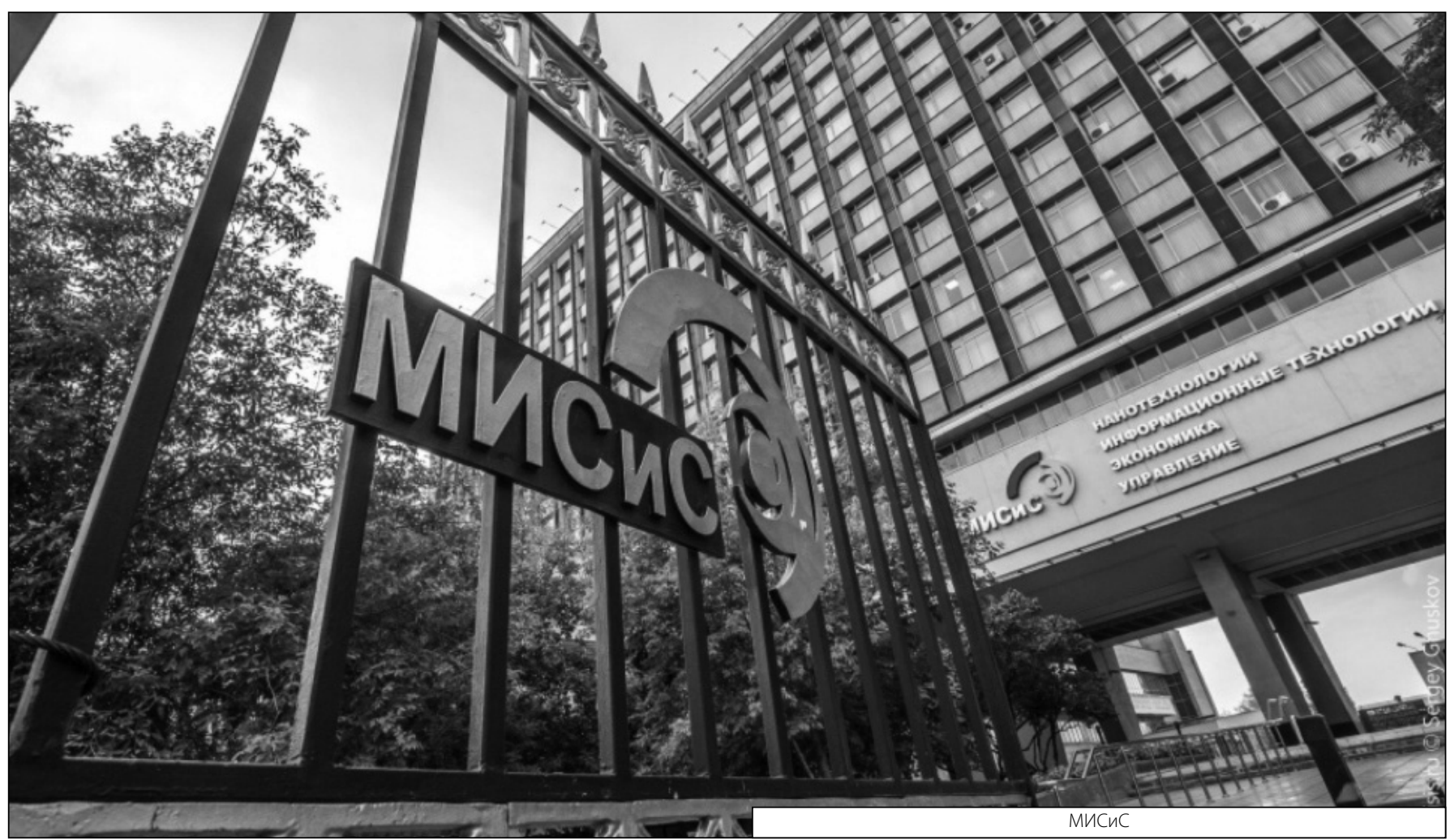

